\title{
On Fox quotients of arbitrary group algebras
}

\author{
Manfred Hart][1] \\ Université de Lille - Nord de France, \\ LAMAV and FR CNRS 2956, \\ ISTV2, UVHC \\ Le Mont Houy, 59313 Valenciennes Cedex 9, France. \\ Email: Manfred.Hartl@univ-valenciennes.fr
}

\begin{abstract}
Certain subquotients of group algebras are determined as a basis for subsequent computations of relative Fox and dimension subgroups. More precisely, for a group $G$ and N-series $\mathcal{G}$ of $G$ let $I_{R, \mathcal{G}}^{n}(G), n \geq 0$, denote the filtration of the group algebra $R(G)$ induced by $\mathcal{G}$, and $I_{R}(G)$ its augmentation ideal. For subgroups $H$ of $G$, left ideals $J$ of $R(H)$ and right $H$-submodules $M$ of $I_{\mathbb{Z}}(G)$ the quotients $I_{R}(G) J / M J$ are studied by homological methods, notably for $M=I_{R}(G) I_{R}(H), I_{R}(H) I_{R}(G)+$ $I([H, G]) R(G)$ and $R(G) I_{R}(N)+I_{R, \mathcal{G}}^{n}(G)$ for a normal subgroup $N$ in $G$; in the latter case the module $I_{R}(G) J / M J$ is completely determined for $n=2$. The groups $I_{\mathbb{Z}, \mathcal{G}}^{n-1}(G) I_{\mathbb{Z}}(H) / I_{\mathbb{Z}, \mathcal{G}}^{n}(G) I_{\mathbb{Z}}(H)$ are studied and explicitly computed for $n \leq 3$ in terms of enveloping rings of certain graded Lie rings and of torsion products of abelian groups.
\end{abstract}

Keywords : group algebra, augmentation quotient, Fox subgroup, N-series, enveloping algebra.

\section{Introduction}

Let $G$ be a group, $R(G)$ be the group algebra of $G$ with coefficients in a commutative ring $R$, and let $I_{R, \mathcal{G}}^{n}(G), n \geq 0$, denote the filtration of $R(G)$ induced by a given N-series $\mathcal{G}$ of $G$, see [21] or section 2 below. In particular, $I_{R, \mathcal{G}}^{0}(G)=$

Research partially supported by an individual fellowship of the Human Capital and Mobility Programme of the European Union during the years 1993-1995.

2000 Mathematical subjects classification 20C07, 20J05, $17 \mathrm{~B} 35$. 
$R(G), I_{R, \mathcal{G}}^{1}(G)$ is the augmentation ideal $I_{R}(G)$ of $R(G)$, and if $\mathcal{G}$ is the lower central series $\gamma$ of $G$, then $I_{R, \gamma}^{n}(G)$ is the $n$-th power $I_{R}^{n}(G)$ of $I_{R}(G)$. As usual, we skip the sub- or superscript $R, \mathcal{G}$ when $R=\mathbb{Z}$ or $\mathcal{G}=\gamma$, resp.

Now let $H$ be a subgroup of $G$ and $J$ be a left ideal of $R(H)$. Then it is a classical problem to study the $n$-th generalized Fox quotient $I_{R, \mathcal{G}}^{n-1}(G) J / I_{R, \mathcal{G}}^{n}(G) J$ for $n \geq 1$ (usually one considers $R=\mathbb{Z}, \mathcal{G}=\gamma$ and $J=I(H)$ ). The first Fox quotient was determined a long time ago [27], and the related quotient $I(G) I(H)^{n-1} / I(G) I(H)^{n}$ was computed by Karan and Vermani [13. But only partial results are known on the second Fox quotient (and some higher ones), for $R=\mathbb{Z}$ and always under some splitting condition, e.g. supposing $H$ to be a semi-direct factor of $G$, see the work of Khambadkone and of Karan and Vermani in [16], [17], 18], 14], [15]. As a striking fact, in all known cases a reduction of the quotient in question was obtained to sums and tensor products among certain subquotients of the group rings of $G$ and $H$ (or of a semidirect complement of $H$ in $G$ ): examples of this phenomenon are given by Proposition 3.4 and by the isomorphisms (11) and (2) below.

In this paper we introduce a homological approach to the study of the quotient $I_{R}(G) J / M J$ for arbitrary $G, H, R, J$ and right $H$-submodules $M$ of $I(G)$, in the same spirit: in certain cases we still find a sum-tensor-decomposition, such as the isomorphisms

$$
\frac{I_{R}(G) J}{I_{R}(G) I_{R}(H) J} \cong \frac{I(H) J}{I^{2}(H) J} \oplus\left(\frac{I(G)}{\mathbb{Z}(G) I(H)}\right) \otimes\left(\frac{J}{I(H) J}\right)
$$

in Theorem 1.5 and

$$
\frac{I_{R}(G) J}{I_{R}(H) I_{R}(G) J+I_{R}([H, G]) R(G) J} \cong\left(\frac{H}{[H, G]} \otimes \frac{J}{I(H) J}\right) \oplus\left(I(G / H) \otimes \frac{J}{I(H) J}\right)
$$

in Theorem 1.10, the latter for normal subgroups $H$. But for general submodules $M$, the group $I_{R}(G) J / M_{R} J$ can only be embedded into a natural exact sequence

$$
\operatorname{Tor}_{1}^{H}\left(\frac{I(G)}{I(H)+M}, J\right) \stackrel{\sigma}{\longrightarrow} \frac{I(H) J}{(I(H) \cap M) J} \stackrel{j}{\longrightarrow} \frac{I_{R}(G) J}{M_{R} J} \longrightarrow \frac{I(G)}{I(H)+M} \otimes_{H} J \longrightarrow 0
$$

thus describing the kernel and cokernel of the canonical map $j$ in terms of a tensor and a torsion product over $H$, see Proposition 1.6. Moreover, the class of the resulting extension $0 \rightarrow \operatorname{Coker}(\sigma) \rightarrow I_{R}(G) J / M_{R} J \rightarrow(I(G) / I(H)+M) \otimes_{H} J \rightarrow$ 0 is also described, in terms of another, more accessible extension.

If $R=Z Z$ and $J=I(H)$ sequence (3) admits a long exact extension, thus describing $\operatorname{Ker}(j)$ in terms of homology groups of $H$ (Theorem 1.7). This also provides a reduction of Fox subgroups of $G$ to induced subgroups of $H$, see Corollary 1.8, for example if $H$ is free then

$$
G \cap(1+M I(H))=H \cap(1+(M \cap I(H)) I(H)) .
$$

A case of particular interest is the quotient $I_{R}(G) J /\left(R(G) I_{R}(N) J+I_{R, \mathcal{G}}^{n}(G) J\right)$ for a normal subgroup $N$ of $G$; when $J=I_{R}(H)$ we call it the Fox polynomial 
group relative to $N$ and $\mathcal{G}$ since it generalizes the polynomial group $P_{n, R}(G)=$ $I_{R}(G) / I_{R}^{n+1}(G)$ introduced by Passi in [20], and its relative version $P_{n, R}(G, N)=$ $I_{R}(G) /\left(I_{R}(N) I_{R}(G)+I_{R}^{n+1}(G)\right)$ used in various contexts, cf. section 2 .

Just as polynomial groups serve to analyze augmentation quotients and, dually, dimension subgroups, we here show how the study of Fox polynomial groups leads to a computation of certain Fox quotients and Fox subgroups. The descriptions obtained formally generalize known results in the case where $H=G$, see Theorems 3.6 and 6.6, they reveal a striking duality between (generalized) Fox polynomial groups and (generalized) Fox subgroups, which comes from the symmetry of pushouts. Fox subgroups are further studied in subsequent work, e.g. in [8]; in this paper we focus on Fox polynomial groups and Fox quotients which we compute in the following cases: If the group $H$ is abelian, we determine the relative Fox polynomial group $I(G) I(H) /\left(\mathbb{Z}(G) I(N) I(H)+I^{n}(G) I(H)\right)$ for all $n$ and normal subgroups $N$ of $G$, in Corollary 2.4. The first generalized Fox quotient is given by

$$
\frac{R(G) J}{I_{R}(G) J} \cong \frac{J}{I(H) J}
$$

see Proposition 3.1 which generalizes a well-known result of Whitcomb for $J=$ $I(H)$. The second generalized Fox quotient is determined in Theorem 3.3, by means of a natural exact sequence, for any subgroup $K$ of $G$ :

$$
\begin{aligned}
& \operatorname{Tor}_{1}^{\mathbb{Z}}\left(\frac{G}{H K G_{(2)}}, \frac{J}{I(H) J}\right) \\
& \downarrow \\
& \frac{I_{R}(H) J}{I_{R}\left(H \cap K G_{(2)}\right) J+I_{R}^{2}(H) J} \quad \stackrel{j}{\longrightarrow} \frac{I_{R}(G) J}{I_{R}(K) J+I_{R, \mathcal{G}}^{2}(G) J} \quad \longrightarrow \quad\left(\frac{G}{H K G_{(2)}}\right) \otimes\left(\frac{J}{I(H) J}\right) \quad \longrightarrow \quad 0
\end{aligned}
$$

A case where the bottom sequence is split short exact is given in Corollary 3.4, this in particular covers the special cases treated in the literature. The above sequence also allows to explicitly compute the intersection $I_{R}(H) J \cap\left(I_{R}(K) J+I_{R}^{2}(G) J\right)$, see Corollary 3.5 .

As to the higher Fox quotients $Q_{n, R}^{\mathcal{G}}(G, H)=I_{R, \mathcal{G}}^{n-1}(G) I_{R}(H) / I_{R, \mathcal{G}}^{n}(G) I_{R}(H)$, Proposition 2.1 provides a natural isomorphism

$$
Q_{n, R}^{\mathcal{G}}(G, H) \cong Q_{n, R}^{\mathcal{G}}(G, \mathbb{1}) \otimes H^{a b}
$$

if $H$ is a free group; if only its $n-1$-step nilpotent quotient is free nilpotent then the right-hand term in (5) must be replaced by a natural quotient, see Theorem 4.1. The resulting description of $Q_{n}(G, H)$ in this case strongly resembles the description of $Q_{2}(G, H)$ for arbitrary subgroups $H$ given in Theorem 3.6.

In order to study the higher Fox quotients $Q_{n}^{\mathcal{G}}(G, H)$ for arbitrary subgroups $H$, we combine our homological methods with a suitable generalization of Quillen's approximation of augmentation quotients [22]. Recall from [21] that for any Nseries $\mathcal{G}$ of $G$ there is a surjective morphism of graded rings

$$
\theta^{\mathcal{G}}: \mathrm{UL}^{\mathcal{G}}(G) \longrightarrow \operatorname{Gr}^{\mathcal{G}}(\mathbb{Z}(G))=\sum_{n \geq 0} I_{\mathcal{G}}^{n}(G) / I_{\mathcal{G}}^{n+1}(G)
$$


where $\mathrm{UL}^{\mathcal{G}}(G)$ is the enveloping algebra of the graded Lie ring $\mathrm{L}^{\mathcal{G}}(G)$ formed by the successive quotients of $\mathcal{G}$. As a key fact, Quillen showes in [22] that rationally $\theta^{\gamma}$ is an isomorphism, i.e. $\operatorname{Ker}\left(\theta^{\gamma}\right)$ is torsion; this is in fact still true for any N-series as follows from fundamental work of Hartley [11], see also [21]. We here extend this construction to Fox quotients by introducing a surjective graded bimodule map

$$
\theta^{\mathcal{G} H}: \mathrm{UL}^{\mathcal{G}}(G) \otimes_{\mathrm{UL}(H)} \overline{\mathrm{UL}}(H) \quad \longrightarrow \quad \sum_{n \geq 1} I_{\mathcal{G}}^{n-1}(G) I(H) / I_{\mathcal{G}}^{n}(G) I(H)
$$

which coincides with $\theta^{\mathcal{G}}$ if $H=G$ and $\mathcal{G}=\gamma$. It turns out that just like $\theta^{\mathcal{G}}$ [4], $\theta^{\mathcal{G H}}$ is an isomorphism in degrees 1 and 2 (Proposition 5.3) but not in higher degrees, in general. We exhibit canonical elements in its kernel (Proposition 5.5); this leads to the problem of whether they generate $\operatorname{Ker}\left(\theta^{\mathcal{G} H}\right)$ modulo torsion, in extension of Quillen's theorem. In degree 3 this is shown to hold, based on the natural exact sequence

$\operatorname{Tor}_{1}^{\mathbb{Z}}\left(G^{A B}, H^{a b}\right) \oplus\left(\operatorname{Ker}\left(l_{2}^{\mathcal{G} H}\right) \cap \operatorname{Ker}\left(c_{2}^{H}\right)\right) \stackrel{\left(\delta_{1}, \delta_{2}\right)}{\longrightarrow} \mathrm{U}_{3}^{\mathcal{G}}(G, H) \stackrel{\theta_{3}^{\mathcal{G} H}}{\longrightarrow} Q_{3}^{\mathcal{G}}(G, H) \rightarrow 0$

established in Theorem 6.1. This amounts to a complete description of the structure of $I_{\mathcal{G}}^{2}(G) I(H) / I_{\mathcal{G}}^{3}(G) I(H)$, both in a functorial way and in terms of a cyclic decomposition of $H^{a b}$ if $H$ is finitely generated. The result obtained resembles our description of $\operatorname{Ker}\left(\theta_{3}^{\mathcal{G}}\right)$ in [4]; the situation here, however, is more intricate as it involves a secondary operator (namely $\delta_{2}$ which is an additive relation with indetermancy $\operatorname{Im}\left(\delta_{1}\right)$ ), a phenomenon which does not occur in the computation of $\operatorname{Ker}\left(\theta_{3}^{\mathcal{G}}\right)$.

The resulting, in fact less complicated description of the group $Q_{3}^{\mathcal{G}}(G, G)$ in Theorem 6.3 and Corollary 6.5 also amounts to a functorial computation of the group

$$
\frac{I^{3}(H) \oplus I([H, K]) I(H)}{I^{4}(H)+I(H) I([H, K]) I(H)+I([H, K, H]) I(H)+I([H, K, K]) I(H)}
$$

taking $H=G$ and a specific N-series $\mathcal{G}$; this group appears in Khambadkone's analysis of Fox quotients of semidirect products [16], but seems not to have been computed yet, not even in special cases.

Finally, we point out that the description of $\operatorname{Ker}\left(\theta_{3}^{\mathcal{G} G}\right)$ above also allows to determine the fourth relative dimension subgroup $G \cap\left(1+I(N) I(G)+I^{4}(G)\right)$ for arbitrary normal subgroups $N$ of $G$ (work in progress).

Conventions. In this paper, the terms of the lower central series of $G$ are denoted by $G_{i}=\left[G_{i-1}, G\right]$, with $G_{1}=G$ and $[a, b]=a b a^{-1} b^{-1}$ for $a, b \in G$. We write $G^{a b}=G / G_{2}$. Maps denoted by $i$ or $j$ (possibly indexed) are always induced by the inclusion $H \hookrightarrow G$ or $I(H) \hookrightarrow I(G)$, and $q$ (possibly indexed) always denotes a canonical quotient map. Moreover, maps denoted by $\mu_{G}, \mu_{G}^{\prime}$ or 
$\mu_{H}, \mu_{H}^{\prime}$ are always induced by multiplication in $\mathbb{Z}(G)$ or $\mathbb{Z}(H)$, respectively, while $\epsilon: R(G) \rightarrow R$ denotes the augmentation map. Maps denoted by $\bar{f}$ defined on some quotient $A / B$ are induced by a homomorphism $f$ on $A$. Tensor products over the ring $\mathbb{Z}(H)$ are denoted by $\otimes_{H}$ and over $\mathbb{Z}$ by $\otimes$.

\section{Contents}

1 Exact sequences for Fox quotients

2 Fox polynomial groups $\quad 14$

3 The first two generalized Fox quotients 18

4 Fox quotients with respect to free nilpotent subgroups $\quad 22$

5 Canonical approximation of Fox quotients 24

6 The third Fox quotient

\section{Exact sequences for Fox quotients}

In this section we provide the key tools (the "pushout lemma" 1.2 and the generic exact sequences 1.4 and (1.6) of our study of the Fox problem in various generalized forms. In particular it allows to deal with Fox quotients and Fox subgroups simultaneously and in a strictly dual way, cf. Theorems 3.6 and 6.6. For general properties of pushouts see [26].

Recall the notation from the introduction. We point out that we throughout use the plain facts that $I_{R}^{n}(G) J=I^{n}(G) J$ for $n \geq 0$, and that $I_{R}(G) \cong I(G) \otimes R$, whence for any subgroup $M$ of $I(G)$,

$$
\frac{I(G)}{M} \otimes J \cong \frac{I(G) \otimes J}{\operatorname{Im}(M \otimes J)} \cong \frac{I_{R}(G) \otimes_{R} J}{\operatorname{Im}\left(M_{R} \otimes_{R} J\right)} \cong \frac{I_{R}(G)}{M_{R}} \otimes_{R} J
$$

where $M_{R}$ is the $R$-submodule of $I_{R}(G)$ generated by $M$. This also implies that $\frac{I(G)}{M} \otimes_{H} J \cong \frac{I_{R}(G)}{M_{R}} \otimes_{R(H)} J$

We start with the following elementary

Lemma 1.1 The canonical map

$$
\mu_{J}: \mathbb{Z}(G) \otimes_{H} J \rightarrow \mathbb{Z}(G) J=R(G) J
$$

is an isomorphism. 
Proof: Using the injections $\eta: J \hookrightarrow R(H) \stackrel{\cong}{\rightarrow} Z(H) \otimes R$ and $\eta^{\prime}: R(G) J \hookrightarrow$ $R(G)$ we have the following commutative square

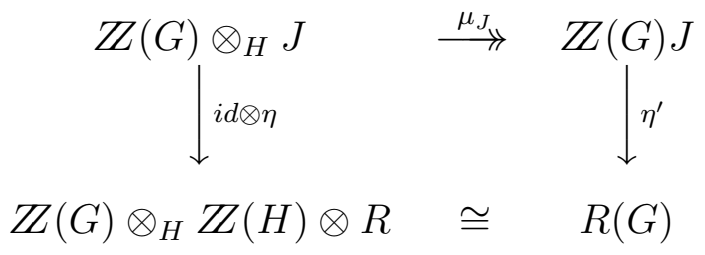

But $i d \otimes \eta$ is injective since $\mathbb{Z}(G)$ is a free $H$-module, so also $\mu_{J}$ is injective.

In order to describe our key lemma, let $U \subset M \subset I(G)$ be right $H$-submodules and $V \subset N \subset J$ be left $R(H)$-submodules such that $U \subset I(H)$. Consider the following commutative square.

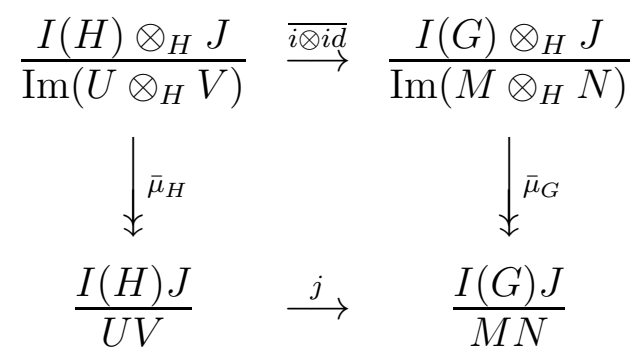

Lemma 1.2 Diagram (6) is a pushout square of $R$-modules, or equivalently, $\operatorname{Ker}\left(\bar{\mu}_{G}\right)=(\overline{i \otimes i d}) \operatorname{Ker}\left(\bar{\mu}_{H}\right)$. This implies that also $\operatorname{Ker}(j)=\bar{\mu}_{H} \operatorname{Ker}(\overline{i \otimes i d})$. Moreover, if $J=I_{R}(H)$ then $\operatorname{Ker}\left(\bar{\mu}_{H}\right)=\pi_{H} \operatorname{Im}\left(\lambda: H_{2}(H, R) \rightarrow I(H) \otimes_{H} I_{R}(H)\right)$ where $\pi_{H}$ is the canonical projection and $\lambda$ is defined in the proof below.

Proof of Lemma 1.2 and Proposition 3.1 below : Consider the following commutative diagram whose middle and bottom row (omitting the right hand bottom corner) are part of the long exact sequences obtained from tensoring with $J$ the short exact sequences $I(K) \stackrel{\nu_{K}}{\longleftrightarrow} \mathbb{Z}(K) \stackrel{\epsilon_{K}}{\longrightarrow} \mathbb{Z}$, for $K=G$ resp. $H$.

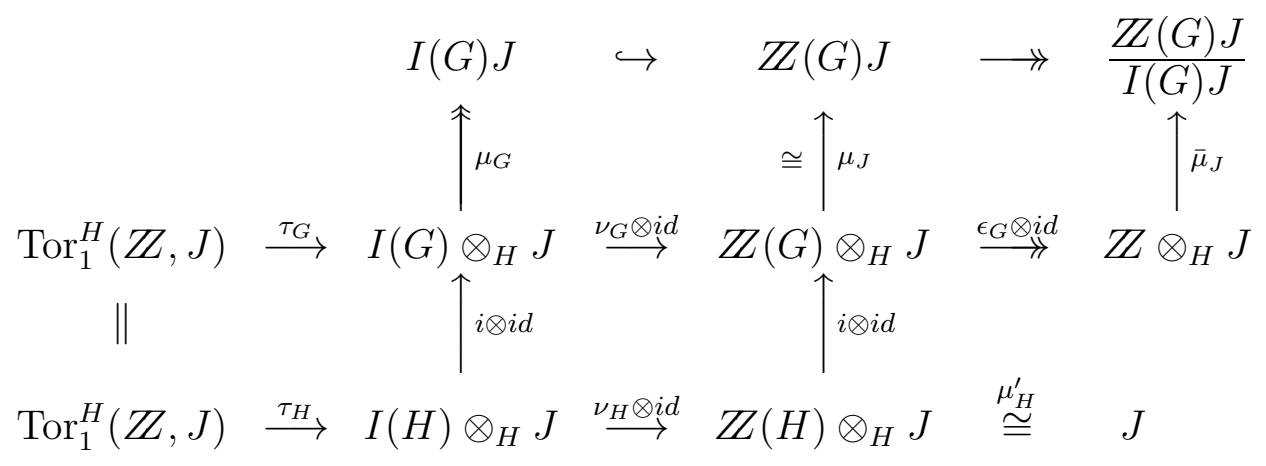


Now $\mu_{J}$ is isomorphic by Lemma 1.1 and $\mu_{G}$ is surjective, so the induced map $\bar{\mu}_{J}$ is an isomorphism, too. But $\mathbb{Z} \otimes_{H} J \cong J / I(H) J$, which proves Proposition 3.1. Furthermore,

$$
\begin{aligned}
\operatorname{Ker}\left(\mu_{G}\right) & =\operatorname{Ker}(\nu \otimes i d)=\operatorname{Im}\left(\tau_{G}\right)=(i \otimes i d) \operatorname{Im}\left(\tau_{H}\right)=(i \otimes i d) \operatorname{Ker}\left(\mu_{H}^{\prime}(\nu \otimes i d)\right) \\
& =(i \otimes i d) \operatorname{Ker}\left(\mu_{H}: I(H) \otimes_{H} J \rightarrow I(H) J\right) .
\end{aligned}
$$

Noting $\pi_{G}: I(G) \otimes_{H} J \rightarrow\left(I(G) \otimes_{H} J\right) / \operatorname{Im}\left(M \otimes_{H} N\right)$ the canonical projection we obtain identities

$$
\begin{aligned}
\operatorname{Ker}\left(\bar{\mu}_{G}\right) & =\pi_{G}\left(\operatorname{Ker}\left(\mu_{G}\right)\right) \\
& =\pi_{G}(i \otimes i d)\left(\operatorname{Ker}\left(\mu_{H}\right)\right) \\
& =(\overline{i \otimes i d}) \pi_{H}\left(\operatorname{Ker}\left(\mu_{H}\right)\right) \\
& =(\overline{i \otimes i d}) \operatorname{Ker}\left(\bar{\mu}_{H}\right) .
\end{aligned}
$$

The identity $\operatorname{Ker}(j)=\bar{\mu}_{H} \operatorname{Ker}(\overline{i \otimes i d})$ now follows by an easy diagram chase using the surjectivity of $\bar{\mu}_{H}$, or more abstractly, by symmetry of pushouts. Now consider the case $J=I_{R}(H)$. First note that for $i \geq 1$, $\operatorname{Tor}_{i}^{H}(Z Z, R(H))=0$ since any resolution $\underline{P}$ of $\mathbb{Z}$ by projective $\mathbb{Z}(H)$-modules is $\mathbb{Z}$-split exact, whence $\underline{P} \otimes_{H} R(H) \cong \underline{P} \otimes_{H} \mathbb{Z}(H) \otimes R \cong \underline{P} \otimes R$ is $R$-split exact. Then dimension shifting along the short exact sequence $I_{R}(H) \hookrightarrow R(H) \stackrel{\epsilon}{\rightrightarrows} R$ of $H$-modules provides a connecting isomorphism $\tau: H_{2}(H, R)=\operatorname{Tor}_{2}^{H}(Z, R) \stackrel{\cong}{\longrightarrow} \operatorname{Tor}_{1}^{H}\left(Z \mathbb{Z}, I_{R}(H)\right)$, so putting $\lambda=\tau_{H} \tau$ also the last part of the assertion is proved.

We first consider an easy but useful case which includes the classical case where $G$ is free.

Corollary 1.3 Suppose that $H$ is a free group with basis $X$. Then for any right $H$-submodule $M$ of $I(G)$ there is an isomorphism

$$
I_{R}(G) I_{R}(H) / M_{R} I_{R}(H) \cong\left(I_{R}(G) / M_{R}\right) \otimes H^{a b}
$$

such that for $x \in X$ and $y \in I_{R}(G)$ the element $\left(y+M_{R}\right) \otimes\left(x H_{2}\right)$ corresponds to $y(x-1)+M_{R} I_{R}(H)$.

Proof: Let $U=0$ and $J=V=N=I_{R}(H)$ in 1.2, As $H$ is free, $H_{2}(H, R)=0$ so $\bar{\mu}_{G}$ is an isomorphism. On the other hand, $I_{R}(H)$ is a free $R(H)$-module with basis $x-1, x \in X$, see [12]. This provides a (non natural) isomorphism of left $H$-modules $I_{R}(H) \cong \mathbb{Z}(H) \otimes H^{a b} \otimes R$. Thus we have isomorphisms $I(G) I_{R}(H) / M I_{R}(H) \cong I(G) \otimes_{H} I_{R}(H) / \operatorname{Im}\left(M \otimes_{H} I_{R}(H)\right) \cong(I(G) / M) \otimes_{H} I_{R}(H)$ $\cong(I(G) / M) \otimes H^{a b} \otimes R \cong\left(I_{R}(G) / M_{R}\right) \otimes H^{a b}$.

Using right-exactness of the tensor product Lemma 1.2 implies the following generic fact which is the basis of all that follows. 
Corollary 1.4 Under the hypothesis of Lemma 1.2 there is a commutative diagram with exact rows where $M^{\prime}=M+I(H) / I(H)$ and $K=\operatorname{Ker}(\overline{i \otimes i d})$.

$$
\begin{aligned}
& K \stackrel{\iota}{\hookrightarrow} \frac{I(H) \otimes_{H} J}{\operatorname{Im}\left(U \otimes_{H} V\right)} \stackrel{\overline{i \otimes i d}}{\longrightarrow} \frac{I(G) \otimes_{H} J}{\operatorname{Im}\left(M \otimes_{H} N\right)} \stackrel{\overline{q \otimes i d}}{\longrightarrow} \frac{(I(G) / I(H)) \otimes_{H} J}{\operatorname{Im}\left(M^{\prime} \otimes_{H} N\right)} \rightarrow 0 \\
& \left\|\bar{\mu}_{H} \quad \downarrow_{\bar{\mu}_{G}} \quad\right\| \\
& K \stackrel{\bar{\mu}_{H \iota}}{\longrightarrow} \quad \frac{I(H) J}{U V} \quad \stackrel{j}{\longrightarrow} \quad \frac{I(G) J}{M N} \quad \stackrel{\overline{\overline{q \otimes i d}}}{\longrightarrow} \frac{(I(G) / I(H)) \otimes_{H} J}{\operatorname{Im}\left(M^{\prime} \otimes_{H} N\right)} \rightarrow 0
\end{aligned}
$$

Consequently, the bottom sequence is induced by the top one, i.e., the abelian group extensions obtained by dividing out the image of $K$ from the left hand terms are such that the top one induces the bottom one via the map induced by $\bar{\mu}_{H}$.

Note that the unhandy terms of the top row of diagram (8) reduce to tensor products when either $U=I(H)$ and $M=I(G)$ or $V=N=J$. We are mainly concerned with the second case in the rest of the paper, but the first one is also of interest: it allows to recover a result of Karan and Vermani [13] from our viewpoint, in a slightly more general form (arbitrary $R$ and $J$ instead of $Z$ and $I^{n-1}(H)$ ).

Theorem 1.5 There is a (non-canonical) isomorphism of $R$-modules

$$
\frac{I_{R}(G) J}{I_{R}(G) I_{R}(H) J} \cong \frac{I(H) J}{I^{2}(H) J} \oplus\left(\frac{I(G)}{Z Z(G) I(H)}\right) \otimes\left(\frac{J}{I(H) J}\right)
$$

Proof: Take $U=I(H), M=I(G), V=N=I(H) J$ in Corollary 1.4, Then we have isomorphisms

$$
\frac{I(H) \otimes_{H} J}{\operatorname{Im}\left(I(H) \otimes_{H} I(H) J\right)} \cong I(H) \otimes_{H} \frac{J}{I(H) J} \cong \frac{I(H)}{I^{2}(H)} \otimes_{H} \frac{J}{I(H) J} \cong \frac{I(H)}{I^{2}(H)} \otimes \frac{J}{I(H) J}
$$

where the first two follow from right-exactness of the tensor product and the latter is due to the fact that both $I(H) / I^{2}(H)$ and $J / I(H) J$ are trivial $H$-modules. Similarly,

$$
\frac{I(G) \otimes_{H} J}{\operatorname{Im}\left(I(G) \otimes_{H} I(H) J\right)} \cong \frac{I(G)}{I(G) I(H)} \otimes \frac{J}{I(H) J} .
$$

Next we recall that the sequence

$$
0 \rightarrow \frac{I(H)}{I^{2}(H)} \stackrel{i_{1}}{\longrightarrow} \frac{I(G)}{I(G) I(H)} \stackrel{q_{1}}{\longrightarrow} \frac{I(G)}{Z Z(G) I(H)} \rightarrow 0
$$


of abelian groups is exact by the classical Fox theorem (i.e. Proposition 3.1 with $J=I(H)$ ). Using this and the above isomorphisms diagram (8) (without the terms $K$ ) identifies with the following diagram where in turn the bottom row is exact and induced by the top row.

$$
\begin{aligned}
& \frac{I(H)}{I^{2}(H)} \otimes \frac{J}{I(H) J} \stackrel{i_{1} \otimes i d}{\longrightarrow} \frac{I(G)}{I(G) I(H)} \otimes \frac{J}{I(H) J} \stackrel{\stackrel{q_{1} \otimes i d}{\longrightarrow}}{\longrightarrow} \frac{I(G)}{Z(G) I(H)} \otimes \frac{J}{I(H) J} \\
& \downarrow h \quad \downarrow g \\
& \frac{I(H) J}{I^{2}(H) J} \quad \stackrel{j_{1}}{\longrightarrow} \quad \frac{I(G) J}{I(G) I(H) J} \quad \stackrel{\underset{q_{1} \otimes i d}{\longrightarrow}}{\longrightarrow} \frac{I(G)}{Z Z(G) I(H)} \otimes \frac{J}{I(H) J}
\end{aligned}
$$

But sequence (9) is split for $I(G) / Z Z(G) I(H)$ is a subgroup of $\mathbb{Z}(G) / Z(G) I(H)$ $\cong \mathbb{Z}(G) \otimes_{H} \mathbb{Z}$ which is a free abelian group since $\mathbb{Z}(G)$ is a free $H$-module. Thus the top sequence in the above diagram is $R$-split short exact, whence so is the bottom sequence, as was to be shown.

In the sequel we study cases of Corollary 1.4 where $V=N=J$. Taking $U=I(H) \cap M$ it amounts to the following description of $I(G) J / M J$ as a group extension.

Proposition 1.6 Let $M$ be a right $H$-submodule of $I(G)$. Then there is a natural commutative diagram of $R$-modules with exact rows where $T=\operatorname{Tor}_{1}^{H}(I(G) /(I(H)+$ $M), J)$ and where the top row is part of the corresponding long exact sequence.

$$
\begin{aligned}
& T \stackrel{\tau}{\longrightarrow} \frac{I(H)}{I(H) \cap M} \otimes_{H} J \stackrel{i \otimes i d}{\longrightarrow} \frac{I(G)}{M} \otimes_{H} J \stackrel{q \otimes i d}{\longrightarrow} \frac{I(G)}{I(H)+M} \otimes_{H} J \rightarrow 0 \\
& \left\|\quad \downarrow^{\mu_{H}} \quad \downarrow^{\mu_{G}}\right\| \\
& T \stackrel{\mu_{H} \tau}{\longrightarrow} \frac{I(H) J}{(I(H) \cap M) J} \quad \stackrel{j}{\longrightarrow} \quad \frac{I_{R}(G) J}{M_{R} J} \quad \stackrel{\overline{q \otimes i d}}{\longrightarrow} \frac{I(G)}{I(H)+M} \otimes_{H} \quad J \quad \rightarrow 0
\end{aligned}
$$

Consequently, the bottom sequence is induced by the top one.

For $R=\mathbb{Z}$ and $J=I(H)$ we can improve Proposition 1.6 as follows.

Theorem 1.7 Let $M \subset I(G)$ be a right $H$-submodule. Then there is a natural long exact sequence 


$$
\begin{aligned}
& \frac{I^{2}(H)}{(I(H) \cap M) I(H)} \stackrel{j}{\rightarrow} \frac{I(G) I(H)}{M I(H)} \stackrel{\tilde{q}}{\rightarrow}\left(\frac{I(G)}{I(H)+M}\right) \otimes_{H} I(H) \quad \rightarrow \quad 0 \\
& \uparrow \mu_{H} \tau_{1} \tau_{2}
\end{aligned}
$$

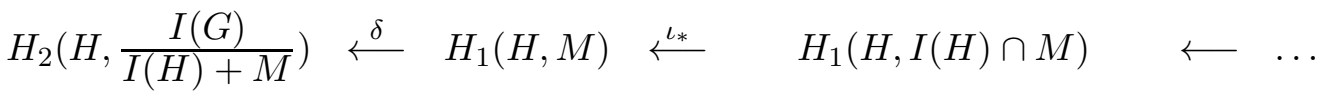

where $\tilde{q}(\overline{x y})=\bar{x} \otimes \bar{y}$ for $x \in I(G), y \in I(H)$, and where the dots "..." stand for extension by the long exact homology sequence induced by the short exact sequence $I(H) \cap M \stackrel{\iota}{\longrightarrow} M \rightarrow M / I(H) \cap M$ of right $H$-modules. The other operators are defined in the proof below.

Note that the homology of $H$ here refers to right coefficient modules, i.e. $H_{i}(H, A)=\operatorname{Tor}_{i}^{H}(A, Z)$ which coincides with usual homology of the opposite module: $\operatorname{Tor}_{i}^{H}(A, \mathbb{Z})=\operatorname{Tor}_{i}^{H}\left(\mathbb{Z}, A^{*}\right)$ where $A^{*}$ is $A$ as an abelian group endowed with the left $H$-action $h \cdot a=a h^{-1}, h \in H, a \in A$.

We point out that the description of the cokernel of $j$ given by the theorem can be used to study Fox quotients while the description of the kernel of $j$ can be used to study Fox subgroups, as follows.

Corollary 1.8 Let $M \subset I(G)$ be a right $H$-submodule. Then

$$
G \cap(1+M I(H))=H \cap\left(1+\pi^{-1} \operatorname{Im}\left(\mu_{H} \tau_{1} \tau_{2}\right)\right)
$$

where $\pi: I^{2}(H) \rightarrow I^{2}(H) /(I(H) \cap M) I(H)$ is the canonical projection.

This means that the study of generalized Fox subgroups $G \cap(1+M I(H))$ is reduced to homology and induced subgroups of $H$, the latter with respect to right ideals in $I(H)$ instead of $I(G)$. In particular we derive the following remarkable fact.

Corollary 1.9 Let $H$ be a free subgroup of $G$ and $M \subset I(G)$ be a right $H$ submodule. Then

$$
G \cap(1+M I(H))=H \cap(1+(I(H) \cap M) I(H)) .
$$

This is immediate from Corollary 1.8 noting that free groups are of cohomological dimension 1. For specific $M$, in particular for $M=I^{n}(G)$, this situation will be further analyzed in subsequent work.

Proof of Theorem 1.7: In Proposition 1.6 take $R=\mathbb{Z}$ and $J=I(H)$, and write $\tau=\tau_{1}$. Now consider the following anticommutative square consisting 
of connecting homomorphisms, where the vertical ones are induced by the short exact sequence $I(H) \stackrel{\nu}{\hookrightarrow} \mathbb{Z}(H) \stackrel{\epsilon}{\longrightarrow} \mathbb{Z}$ and the bottom one by the sequence $I(H) / I(H) \cap M \hookrightarrow I(G) / M \rightarrow I(G) / I(H)+M$.

$$
\begin{array}{ccc}
\operatorname{Tor}_{1}^{H}(I(G) /(I(H)+M), I(H)) & \stackrel{\tau_{1}}{\longrightarrow}(I(H) / I(H) \cap M) \otimes_{H} I(H) \\
\cong \uparrow \tau_{2} & \uparrow \tau_{2}^{\prime} \\
\operatorname{Tor}_{2}^{H}(I(G) /(I(H)+M), \mathbb{Z}) & \stackrel{\tau_{1}^{\prime}}{\longrightarrow} & \operatorname{Tor}_{1}^{H}(I(H) / I(H) \cap M, \mathbb{Z})
\end{array}
$$

Here $\tau_{2}$ is an isomorphism since $\operatorname{Tor}_{i}^{H}(-, \mathbb{Z}(H))=0$ for $i \geq 1$ as $\mathbb{Z}(H)$ is a free $H$-module. Hence $\operatorname{Ker}(j)=\operatorname{Im}\left(\mu_{H} \tau_{1} \tau_{2}\right)=\operatorname{Im}\left(\mu_{H} \tau_{2}^{\prime} \tau_{1}^{\prime}\right)$. So it remains to exhibit $\operatorname{Ker}\left(\mu_{H} \tau_{2}^{\prime} \tau_{1}^{\prime}\right)$. Consider the following commutative diagram of right $H$-modules where $U=I(H) \cap M$ and $\epsilon^{\prime}(\bar{x})=\overline{x-\epsilon(x)}$ for $x \in \mathbb{Z}(G)\left(\epsilon^{\prime}\right.$ is $H$-linear as for $h \in H, x h-\epsilon(x h)=(x-\epsilon(x)) h+\epsilon(x)(h-1) \equiv(x-\epsilon(x)) h \bmod I(H))$.

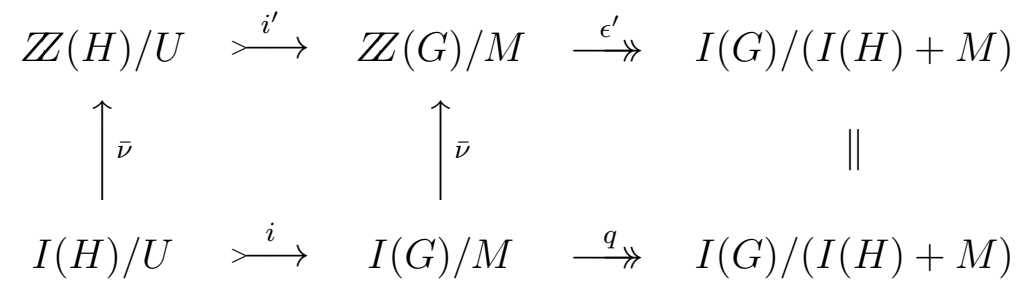

The rows are short exact, so applying the functor $\operatorname{Tor}_{1}^{H}(-, Z Z)$ provides the first two commutative squares of the following diagram with exactness in the second term from the left.

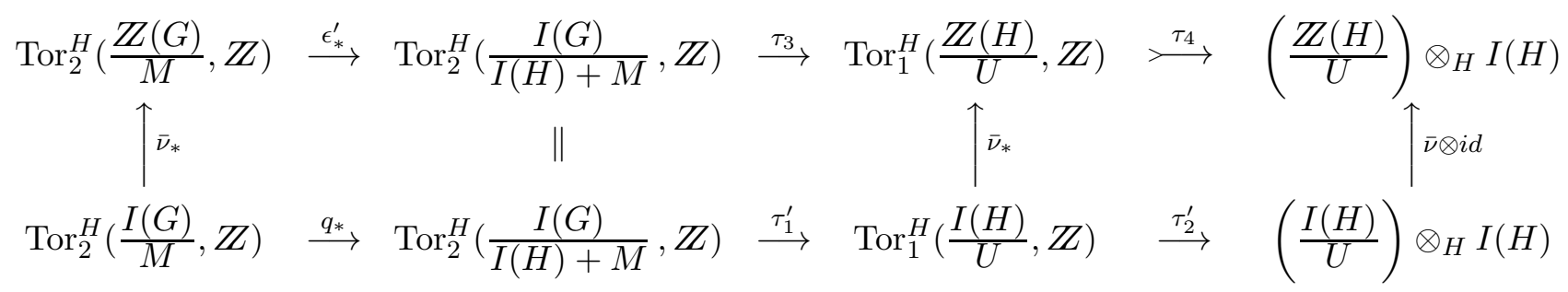

The operators $\tau_{2}^{\prime}$ and $\tau_{4}$ are injective as they are part of the corresponding long exact sequences induced by the short exact sequence $I(H) \stackrel{\nu}{\longrightarrow} \mathbb{Z}(H) \stackrel{\epsilon}{\longrightarrow} \mathbb{Z}$, and as $\operatorname{Tor}_{1}^{H}(-, \mathbb{Z}(H))=0$. Now under the isomorphisms $(\mathbb{Z}(H) / U) \otimes_{H} I(H) \cong$ $\mathbb{Z}(H) \otimes_{H} I(H) / \operatorname{Im}\left(U \otimes_{H} I(H)\right) \cong I(H) / U I(H)$ the map $(\bar{\nu} \otimes i d)$ corresponds to $\mu_{H}$, so that $\operatorname{Ker}\left(\mu_{H} \tau_{2}^{\prime} \tau_{1}^{\prime}\right)=\operatorname{Ker}\left((\bar{\nu} \otimes i d) \tau_{2}^{\prime} \tau_{1}^{\prime}\right)=\operatorname{Ker}\left(\tau_{4} \tau_{3}\right)=\operatorname{Ker}\left(\tau_{3}\right)=\operatorname{Im}\left(\epsilon_{*}^{\prime}\right)$. To compute $\operatorname{Ker}\left(\epsilon^{\prime}\right)$ we consider the canonical extension to the left of the top row of the foreging diagram, and we use the connecting homomorphisms induced by the short exact sequences $U \hookrightarrow \mathbb{Z}(H) \rightarrow \mathbb{Z}(H) / U$ and $M \hookrightarrow \mathbb{Z}(G) \rightarrow \mathbb{Z}(G) / M$ 
which are isomorphisms as $Z(H)$ and $Z(G)$ are free $H$-modules.

$$
\begin{array}{ccc}
\operatorname{Tor}_{1}^{H}(U, \mathbb{Z}) & \stackrel{\iota_{*}}{\longrightarrow} & \operatorname{Tor}_{1}^{H}(M, Z Z) \\
\cong \uparrow \tau_{5} & \cong \uparrow \tau_{6} \\
\operatorname{Tor}_{2}^{H}\left(\frac{Z Z(H)}{U}, \mathbb{Z}\right) & \stackrel{i_{*}^{\prime}}{\longrightarrow} \operatorname{Tor}_{2}^{H}\left(\frac{\mathbb{Z}(G)}{M}, \mathbb{Z}\right) \stackrel{\epsilon_{*}^{\prime}}{\longrightarrow} \operatorname{Tor}_{2}^{H}\left(\frac{I(G)}{I(H)+M}, \mathbb{Z}\right) .
\end{array}
$$

So putting $\delta=\epsilon_{*}^{\prime} \tau_{6}^{-1}$ the theorem is proved.

We still give another application of Proposition [1.6 which is motivated by the computation of the induced subgroup $G \cap(1+I(H) I(G) I(H)+I([H, G]) I(H))$ by Tahara, Vermani and Razdan in 25]; we show that their result can be recovered and generalized to arbitrary $R$ by the computation of a related quotient of $I_{R}(G)$, as follows.

Theorem 1.10 Suppose that $H$ is normal in $G$. Then there is a non-natural isomorphism of $R$-modules

$\frac{I_{R}(G) J}{I_{R}(H) I_{R}(G) J+I_{R}([H, G]) R(G) J} \cong\left(\frac{H}{[H, G]} \otimes \frac{J}{I(H) J}\right) \oplus\left(I(G / H) \otimes \frac{J}{I(H) J}\right)$

as well as identities

$$
\begin{aligned}
G & \cap\left(1+I_{R}(H) I_{R}(G) I_{R}(H)+I_{R}([H, G]) I_{R}(H)\right) \\
& =G \cap\left(1+I_{R}(H) I_{R}(G) I_{R}(H)+I_{R}([H, G]) R(G) I_{R}(H)\right) \\
& =H \cap\left(1+I_{R}([H, G]) I_{R}(H)+I_{R}^{3}(H)\right) .
\end{aligned}
$$

We point out that the latter term can be easily made explicit by using our formula in [8] for the second relative dimension subgroup $G \cap\left(1+I_{R}(K) I_{R}(G)+\right.$ $I_{R}^{3}(G)$ ) for all $K \leq G$ and $R$ (reproved differently for $R=\mathbb{Z}$ in [25]); as the result is rather involved for arbitrary $R$ we refrain from stating it here.

Proof: In Proposition [1.6, take $M=I(H) I(G)+I([H, G]) Z Z(G)$ and $V=N=$ $J$. Let $N$ a normal subgroup of $G$. Then there is a canonical isomorphism of right $G$-modules

$$
\Psi_{N}: I(G) / I(N) \mathbb{Z}(G) \rightarrow I(G / N), \quad \overline{a-1} \mapsto a N-1
$$

In particular, the map $\Psi_{[H, G]}$ induces another isomorphism of right $G$-modules $\gamma: I(G) / M \stackrel{\cong}{\longrightarrow} I(G /[H, G]) / I(H /[H, G]) I(G /[H, G])$. Furthermore, $N$ gives 
rise to the following exact sequence of left $G / N$-modules where $D(\bar{a})=\overline{a-1}$ for $a \in H$, see Theorem VI.6.3 in [12].

$$
0 \rightarrow N^{a b} \stackrel{D}{\longrightarrow} \frac{I(G)}{I(N) I(G)} \stackrel{\overline{I\left(q^{\prime}\right)}}{\longrightarrow} I(G / N) \rightarrow 0 .
$$

In particular, taking $N=G$ one gets the wellknown isomorphism of groups

$$
\phi_{G}: G / G_{2} \cong I(G) / I^{2}(G), \quad \phi_{G}\left(a G_{2}\right)=a-1+I^{2}(G) \text { for } a \in G .
$$

Taking $N=[H, G]$ we obtain the following commutative diagram with exact rows where $U=I(H) \cap M$.

$$
\begin{aligned}
& 0 \rightarrow \frac{I(H)}{U} \quad \stackrel{i}{\rightarrow} \quad \frac{I(G)}{M} \quad \stackrel{q}{\rightarrow} \frac{I(G)}{I(H)+M} \rightarrow 0 \\
& \downarrow \tilde{\gamma} \quad \cong \downarrow \gamma \quad \cong \downarrow \Psi_{H} \\
& 1 \rightarrow H /[H, G] \stackrel{D}{\rightarrow} \frac{I(G /[H, G])}{I(H /[H, G]) I(G /[H, G])} \stackrel{\overline{I\left(q^{\prime}\right)}}{\longrightarrow} \quad I(G / H) \quad \rightarrow \quad 0
\end{aligned}
$$

Here $\tilde{\gamma}$ is induced by $\gamma$ and hence is an isomorphism, too. The bottom sequence is $Z$-split as $I(G / H)$ is a free $Z$-module; hence so is the top sequence. Moreover, the right $H$-action on each of its terms is trivial; for $I(H) / U$ and $I(G) / M+I(H)$ this follows from the inclusions $I^{2}(H) \subset U$ and $\mathbb{Z}(G) I(H)=$ $I(H) \mathbb{Z}(G) \subset M+I(H)$, resp., and for $I(G) / M$ by right $G$-linearity of $\gamma$ and the fact that $H /[H, G]$ is central in $G /[H, G]$, whence $I(H /[H, G]) I(G /[H, G])=$ $I(G /[H, G]) I(H /[H, G])$. Using these facts diagram (10) (without the terms $T$ ) identifies with the following diagram.

$$
\begin{aligned}
& 0 \rightarrow \quad \frac{I(H)}{U} \otimes \frac{J}{I(H) J} \quad \stackrel{i \otimes i d}{\longrightarrow} \quad \frac{I(G)}{M} \otimes \frac{J}{I(H) J} \quad \stackrel{q \otimes i d}{\longrightarrow} \quad I(G / H) \otimes \frac{J}{I(H) J} \quad \rightarrow \quad 0 \\
& \downarrow \downarrow \bar{\mu}_{H} \quad \| \\
& 0 \rightarrow \frac{I(H) J}{I([H, G]) J+I^{2}(H) J} \stackrel{j}{\rightarrow} \frac{I(G) J}{I(H) I(G) J+I([H, G]) \mathbb{Z}(G) J} \stackrel{\overline{q \otimes i d}}{\longrightarrow} I(G / H) \otimes \frac{J}{I(H) J} \quad \rightarrow \quad 0
\end{aligned}
$$

By the foregoing the top row is $R$-split exact, whence so is the bottom row by Proposition 1.6 which implies the desired isomorphism. Now take $J=I_{R}(H)$. We 
claim that the following relations hold.

$$
\begin{aligned}
G & \cap\left(1+I_{R}(H) I_{R}(G) I_{R}(H)+I_{R}([H, G]) I_{R}(H)\right) \\
& \subset G \cap\left(1+I_{R}(H) I_{R}(G) I_{R}(H)+I_{R}([H, G]) R(G) I_{R}(H)\right) \\
& =H \cap\left(1+I_{R}(H) I_{R}(G) I_{R}(H)+I_{R}([H, G]) R(G) I_{R}(H)\right) \\
& =H \cap\left(1+I_{R}([H, G]) I_{R}(H)+I_{R}^{3}(H)\right)
\end{aligned}
$$

Indeed, (14) being plain, (15) follows from the relation $G \cap\left(1+I_{R}(G) I_{R}(H)\right)$ $=H \cap\left(1+I_{R}^{2}(H)\right)$ (cf. Corollary 3.2 below), which also implies (16) by injectivity of the map $j$ in the above diagram. As the last term is contained in the first the identities of induced subgroups given in the assertion are proved.

\section{Fox polynomial groups}

Polynomial groups $P_{n, R}(G)=I_{R}(G) / I_{R}^{n+1}(G)$ were introduced by Passi in [20] (see also [21]), along with a notion of polynomial maps from groups to $R$-modules such that the map $p_{n, R}: G \rightarrow P_{n, R}(G), p_{n, R}(a)=a-1+I_{R}^{n+1}(G)$ is universal polynomial of degree $\leq n$. Relative polynomial groups $P_{n, R}(G, N)=$ $I_{R}(G) /\left(I_{R}(N) I_{R}(G)+I_{R}^{n+1}(G)\right)$ for normal subgroups $N$ were formally introduced in [5] but had been implicitely studied in the literature before. Indeed, these constructions proved to be very useful in the study of dimension subgroups $D_{n, R}(G):=G \cap\left(1+I_{R}^{n}(G)\right)=\operatorname{Ker}\left(p_{n-1, R}\right)$ as well as of augmentation quotients $Q_{n, R}(G):=I_{R}^{n}(G) / I_{R}^{n+1}(G)=\operatorname{Im}\left(\left(G^{a b}\right)^{\otimes n} \otimes R \rightarrow P_{n, R}(G)\right)$, see [21] and [4]; moreover, they are used in [5] to study extensions of torsionfree nilpotent groups and in [6] to determine the Schur multiplier of 2-step nilpotent groups. We here extend the approach of augmentation quotients via polynomial groups to Fox quotients $Q_{n, R}(G, H)=I_{R}^{n-1}(G) I_{R}(H) / I_{R}^{n}(G) I_{R}(H)$ : first consider relative Fox polynomial groups

$$
\begin{aligned}
& P_{n, R}(G, N ; H)=R(G) I_{R}(H) /\left(R(G) I_{R}(N) I_{R}(H)+I_{R}^{n}(G) I_{R}(H)\right) \\
& \bar{P}_{n, R}(G, N ; H)=I_{R}(G) I_{R}(H) /\left(R(G) I_{R}(N) I_{R}(H)+I_{R}^{n}(G) I_{R}(H)\right) ;
\end{aligned}
$$

note that $P_{n, R}(G, N)=P_{n, R}(G, N ; G)$ and $P_{n, R}(G, N)^{2}=\bar{P}_{n, R}(G, N ; G)$. In a second step determine $Q_{n, R}(G, H)$ as a subgroup of $\bar{P}_{n, R}(G, \mathbb{1} ; H)$. We here carry out this program for $n=2$ and all $R$ and for $n=3$ and $R=\mathbb{Z}$.

Actually we consider a more general version of $\bar{P}_{n, R}(G, K ; H)$, replacing $I_{R}(H)$ by $J$ and the augmantation powers $I_{R}^{n}(G)$ by the filtration terms $I_{R, \mathcal{G}}^{n}(G)$ induced by an N-series $\mathcal{G}$ of $G$. This degree of generality is necessary in many contexts, such as polynomial cohomology [5] or (ordinary) augmentation and Fox quotients 
and subgroups of semidirect products [24], [16], and even of arbitrary groups: we show in subsequent work that under suitable conditions the classical Fox subgroup $G \cap\left(1+I^{n}(G) I(H)\right)$ equals $H \cap\left(1+I_{\mathbb{Z}, \mathcal{H}}^{n}(H) I(H)\right)$ for an appropriate N-series $\mathcal{H}$ of $H$.

Let us recall the necessary definitions. An $N$-series $\mathcal{G}$ of $G$ is a descending chain of subgroups

$$
G=G_{(1)} \supset G_{(2)} \supset G_{(3)} \supset \ldots
$$

such that $\left[G_{(i)}, G_{(j)}\right] \subset G_{(i+j)}$ for $i, j \geq 1$. A given N-series $\mathcal{G}$ induces a descending chain of two-sided ideals of $R(G)$

$$
R(G)=I_{R, \mathcal{G}}^{0}(G) \supset I_{R, \mathcal{G}}^{1}(G) \supset I_{R, \mathcal{G}}^{2}(G) \supset \ldots
$$

by defining $I_{R, \mathcal{G}}^{n}(G)$ (for $n \geq 1$ ) to be the $R$-submodule of $R(G)$ generated by the elements

$$
\left(a_{1}-1\right) \cdots\left(a_{r}-1\right), \quad r \geq 1, a_{i} \in N_{k_{i}}, \text { such that } k_{1}+\ldots+k_{r} \geq n .
$$

For example, the following N-series frequently appear in the literature:

- the lower central series, denoted by $\gamma=\gamma_{G}$, where $I_{R, \gamma}^{n}(G)=I_{R}^{n}(G)$;

- the series $\sqrt{\gamma}$ defined by $G_{(i)}=\sqrt{G_{i}}$, the isolator of $G_{i}$; here $I_{\mathbb{Z}, \sqrt{\gamma}}^{n}(G)$ is the isolator of $I^{n}(G)$, see [5];

- if $G$ itself is a subgroup of a group $\Gamma$, the N-series defined by $G_{(i)}=G \cap \Gamma_{i}$ or $G_{(i)}=\left[G_{(i-1)}, \Gamma\right]$ if $G$ is normal in $\Gamma$, see [3], [24].

In the sequel we add a supercript $\mathcal{G}$ to the terms defined above (writing $P_{n, R}^{\mathcal{G}}(G), P_{n, R}^{\mathcal{G}}(G, N ; H)$ etc.) when we replace $I_{R}^{k}(G), k=n-1, n, n+1$, by $I_{R, \mathcal{G}}^{k}(G)$ in their definition; and the super- or subscripts $R, \mathcal{G}$ are frequently suppressed from the notation when $R=\mathbb{Z}$ or $\mathcal{G}=\gamma$, resp.

Actually the pushout lemma and its corollary in section 1 provide the key tools for studying these notions: it turns out that the isomorphism $Q_{2}^{\mathcal{G}}(G)=$ $P_{2}^{\mathcal{G}}(G)^{2}+p_{2}\left(G_{(2)}\right) \cong \mathrm{U}_{2} \mathrm{~L}^{\mathcal{G}}(G)$ and its analogon for $P_{3}^{\mathcal{G}}(G)^{2}+p_{2}\left(G_{(3)}\right)$ in [4] formally generalize to the Fox case once written in form of a pushout, see Theorems 3.6 and 6.6 below. At the same time the method provides a strictly dual approach to Fox subgroups which is pursued in [8] to determine the second relative Fox subgroup over any ring of coefficients.

We start by an easy case, noting that there is a canonical approximation of the Fox quotient $Q_{n}^{\mathcal{G}}(G, H)=I_{\mathcal{G}}^{n-1}(G) I(H) / I_{\mathcal{G}}^{n}(G) I(H)$, namely by means of the natural surjective homomorphism

$$
\zeta_{n}^{\mathcal{G}}: Q_{n-1}^{\mathcal{G}}(G) \otimes H^{a b} \rightarrow Q_{n}^{\mathcal{G}}(G, H)
$$


where $\zeta_{n}\left(\left(x+I_{\mathcal{G}}^{n}(G)\right) \otimes h H_{2}\right)=x(h-1)+I_{\mathcal{G}}^{n}(G) I(H)$ for $x \in I_{\mathcal{G}}^{n-1}(G), h \in H$. This map rarely is an isomorphism, but it is at least in the following case.

Let $\overline{\mathcal{G}}$ be the N-series of $G / N$ given by the image of $\mathcal{G}$ under the canonical projection.

Proposition 2.1 If $H$ is a free group then there are isomorphisms

$$
\begin{aligned}
\bar{P}_{n, R}^{\mathcal{G}}(G, N ; H) & \cong P_{n-1, R}^{\overline{\mathcal{G}}}(G / N) \otimes H^{a b} \\
Q_{n, R}^{\mathcal{G}}(G, H) & \cong Q_{n-1, R}^{\mathcal{G}}(G) \otimes H^{a b}
\end{aligned}
$$

the first of which is non natural while the second one is given by $\zeta_{n}^{\mathcal{G}}$.

Proof: The first isomorphism is immediate from Corollary 1.3 by taking $M=$ $\mathbb{Z}(G) I(N)+I^{n}(G)$ and using the isomorphism $\Psi_{N}$ in (11). Then the composite $\operatorname{map} Q_{n-1, R}^{\mathcal{G}}(G) \otimes H^{a b} \stackrel{\zeta_{n}^{\mathcal{G}}}{\longrightarrow} Q_{n}^{\mathcal{G}}(G, H) \hookrightarrow \bar{P}_{n, R}^{\mathcal{G}}(G,\{1\} ; H) \cong P_{n-1, R}^{\mathcal{G}}(G) \otimes H^{a b}$ equals $i \otimes i d$ with $i: Q_{n-1, R}^{\mathcal{G}}(G) \hookrightarrow P_{n-1, R}^{\mathcal{G}}(G)$. But $i \otimes i d$ is injective as $H^{a b}$ is a free $Z Z$-module, hence so is $\zeta_{n}^{\mathcal{G}}$.

For a refinement of this fact for free nilpotent groups $H$ see section 4 below.

To study the structure of $I_{R}(G) J /\left(R(G) I_{R}(N) J+I_{R, \mathcal{G}}^{n}(G) J\right)$ for arbitrary $H$ we use Lemma 1.2 for $M=I(N) \mathbb{Z}(G)+I_{\mathcal{G}}^{n}(G)$ and $U$ being some right $H$-submodule of $I(H)$ and $M$ containing $I^{n}(H)$; one may take $U$ equal to $I^{n}(H)$, cf. the proofs of corollaries 2.4, 3.6 and Theorem 6.6, or equal to $I(H \cap N) \mathbb{Z}(H)+I^{n}(H)$ where $I(H) / U \cong P_{n-1}(H N / N)$, or equal to $I(H) \cap M$, cf. Proposition 2.3. Now consider the following commutative square.

$$
\begin{aligned}
& \left(\frac{I(H)}{U}\right) \otimes_{H}\left(\frac{J}{I^{n-1}(H) J}\right) \stackrel{i \otimes i d}{\longrightarrow} P_{n-1}^{\overline{\mathcal{G}}}(G / N) \otimes_{H}\left(\frac{J}{I^{n-1}(H) J}\right) \\
& \downarrow \mu_{H} \quad \downarrow \mu_{G} \\
& \frac{I_{R}(H) J}{U_{R} J} \quad \stackrel{j}{\longrightarrow} \quad \frac{I_{R}(G) J}{R(G) I_{R}(N) J+I_{R, \mathcal{G}}^{n}(G) J}
\end{aligned}
$$

Proposition 2.2 Diagram (18) is a pushout square of abelian groups; in other words, the map $\mu_{G}$ induces a natural isomorphism

$$
\frac{I_{R}(G) J}{R(G) I_{R}(N) J+I_{R, \mathcal{G}}^{n}(G) J} \cong P_{n-1}^{\overline{\mathcal{G}}}(G / N) \otimes_{H}\left(\frac{J}{I^{n-1}(H) J}\right) /(i \otimes i d) \operatorname{Ker}\left(\mu_{H}\right) .
$$

This also implies the relation

$$
\operatorname{Ker}(j)=\mu_{H} \operatorname{Ker}(i \otimes i d) .
$$


Proof: This is a special case of Lemma 1.2, Take $V=N=J$ in (6). Then right-exactness of the tensor product provides isomorphisms

$$
\frac{I(G) \otimes_{H} J}{\operatorname{Im}\left(M \otimes_{H} J\right)} \cong\left(\frac{I(G)}{M}\right) \otimes_{H} J \cong\left(\frac{I(G)}{M}\right) \otimes_{H}\left(\frac{J}{I^{n-1}(H) J}\right)
$$

similarly for $G$ being replaced by $H$. Using this it is easy to identify diagram (18) with diagram (6), so Lemma 1.2 gives the result.

Proposition 1.6 here takes the following form.

Corollary 2.3 For $M=I(N) \mathbb{Z}(G)+I_{\mathcal{G}}^{n}(G)$ there is a natural commutative diagram with exact rows where we abbreviate $U=I(H) \cap M, J_{n-1}=J / I^{n-1}(H) J$, $T=\operatorname{Tor}_{1}^{H}\left(I(G) /(I(H)+M), J_{n-1}\right), \bar{H}=H N / N$ and where the top row is part of the corresponding long exact sequence.

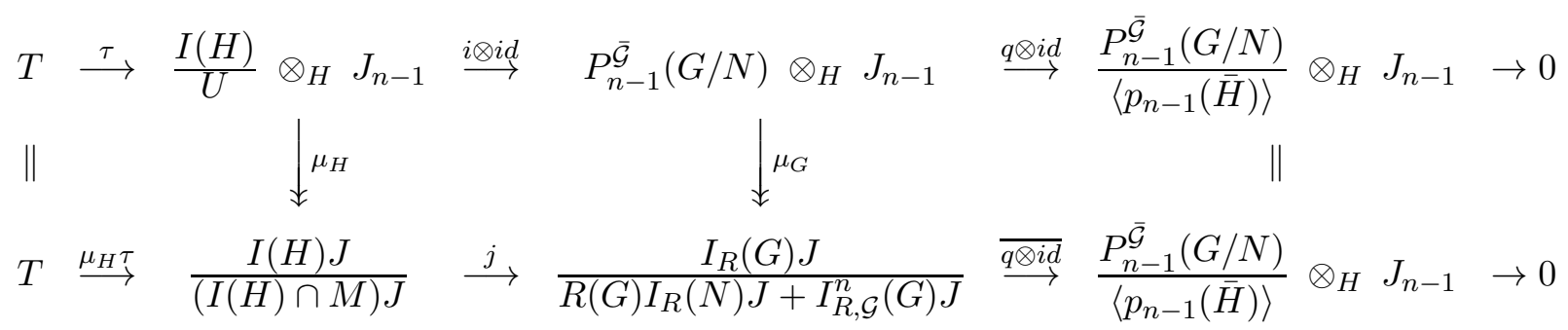

Consequently, the bottom sequence is induced by the top one.

Corollary 2.4 (i) There is a natural exact sequence

$$
H_{2}(H, R) \stackrel{\tilde{\lambda}}{\longrightarrow} P_{n-1}^{\overline{\mathcal{G}}}(G / N) \otimes_{H} P_{n-1, R}(H) \stackrel{\mu_{G}}{\longrightarrow} \bar{P}_{n, R}^{\mathcal{G}}(G, N ; H) \rightarrow 0 .
$$

(ii) If $H$ is abelian and $R=\mathbb{Z}$, sequence (20) becomes

$$
H \wedge H \stackrel{\lambda^{\prime}}{\longrightarrow} P_{n-1}^{\overline{\mathcal{G}}}(G / N) \otimes_{H} P_{n-1}(H) \stackrel{\mu_{G}}{\longrightarrow} \bar{P}_{n}(G, N ; H) \rightarrow 0
$$

where $H \wedge H=H \otimes H /\langle\{h \otimes h \mid h \in H\}\rangle$ and $\lambda^{\prime}\left(h \wedge h^{\prime}\right)=p_{n-1}(h) \otimes p_{n-1}\left(h^{\prime}\right)-$ $p_{n-1}\left(h^{\prime}\right) \otimes p_{n-1}(h)$.

(iii) Let $H=\langle t\rangle$ be cyclic of order $m$. Recall the norm element $N(t)=1+t+$ $\ldots+t^{m-1}$ and let $\bar{N}(t)$ be its image in $\mathbb{Z}(G / N)$. Then there is an isomorphism

$$
\gamma: \bar{P}_{n}(G, N ; H) \cong P_{n-1}^{\overline{\mathcal{G}}}(G / N) / P_{n-1}^{\overline{\mathcal{G}}}(G / N) \bar{N}(t)
$$

which sends the coset of $x y, x \in I(G)$ and $y \in I(H)$, to the coset of $x y^{\prime}$ where $y^{\prime} \in \mathbb{Z}(H)$ such that $y=y^{\prime}(t-1)$. 
Proof : Defining $\tilde{\lambda}=(i \otimes i d) \lambda$ assertion (i) follows from Lemma 1.2. Then part (ii) is an immediate consequence of the wellknown computation $H_{2}(H) \cong H \wedge H$ for abelian groups $H$. Now let $H=\langle t\rangle$ be of order $m$. Then there is an $H$-linear isomorphism $\mathbb{Z}(H) / \mathbb{Z}(H) N(t) \stackrel{\cong}{\longrightarrow} I(H)$ sending $\bar{x}$ to $x(t-1)$ for $x \in \mathbb{Z}(H)$. As here $H \wedge H=0$ one gets isomorphisms

$$
\begin{aligned}
\bar{P}_{n}(G, N ; H) & \cong P_{n-1}^{\overline{\mathcal{G}}}(G / N) \otimes_{H} P_{n-1}(H) \\
& \cong P_{n-1}^{\overline{\mathcal{G}}}(G / N) \otimes_{H} I(H) \\
& \cong P_{n-1}^{\overline{\mathcal{G}}}(G / N) \otimes_{H}(\mathbb{Z}(H) / \mathbb{Z}(H) N(t)) \\
& \cong\left(P_{n-1}^{\overline{\mathcal{G}}}(G / N) / P_{n-1}^{\overline{\mathcal{G}}}(G / N) \cdot \mathbb{Z}(H) N(t)\right) \otimes_{H} \mathbb{Z}(H) \\
& \cong P_{n-1}^{\overline{\mathcal{G}}}(G / N) / P_{n-1}^{\overline{\mathcal{G}}}(G / N) \bar{N}(t)
\end{aligned}
$$

where the dot . denotes the canonical right $H$-module structure of $P_{n-1}^{\overline{\mathcal{G}}}(G / N)$.

We remark that parts (ii) and (iii) of the preceding corollary can easily be generalized to arbitrary coefficient rings $R$ by using part (i) and the universal coefficient sequence, along with the explicit generators of $\operatorname{Tor}_{1}^{\mathbb{Z}}\left(H^{a b}, R\right)$ provided in [19] V.6. We leave it to the interested reader to write out the details.

\section{The first two generalized Fox quotients}

We first quote the following elementary fact which was first proved by Whitcomb for $J=I(H)$ in [27].

Proposition 3.1 The first generalized Fox quotient is reduced to the coinvariants of $J$ by the natural isomorphism

$$
\frac{R(G) J}{I_{R}(G) J} \cong \frac{J}{I(H) J}
$$

This may be well-known but was reproved anyway together with Lemma 1.2.

Corollary 3.2 If $J \subset I_{R}(H)$ one has the relation

$$
G \cap\left(1+I_{R}(G) J\right)=H \cap\left(1+I_{R}(H) J\right) .
$$

This was proved for $R=\mathbb{Z}$ in [13].

Proof: We know from [7] that

$$
G \cap\left(1+I_{R}(G) J\right)=H \cap\left(1+I_{R}(H) J\right) .
$$


Now let $T$ be a left transversal for $H$ in $G$ containing 1. Then $R(G)=\bigoplus_{t \in T} t . R(H)$ and $R(G) J=\bigoplus_{t \in T} t . J \subset \bigoplus_{t \in T} t . R(H)$. As $I_{R}(H)$ lies in the summand 1.R $(H)$ we see that $I_{R}(H) \cap R(G) J=J$, whence

$$
H \cap(1+R(G) J)=H \cap(1+J) .
$$

The assertion now follows combining (21), (22) and Proposition 3.1 .

Now we turn to the second generalized Fox quotient. Let $K$ be some subgroup of $G$. Take $n=2$ and $N=K G_{(2)}$ in Corollary 2.3, noting that then $M=$ $\mathbb{Z}(G) I\left(K G_{(2)}\right)+I_{\mathcal{G}}^{2}(G)=I(K)+I_{\mathcal{G}}^{2}(G)=I\left(K G_{(2)}\right)+I^{2}(G)$. All tensor products over $H$ reduce to tensor products over the integers as all terms involved are trivial $H$-modules. Another reduction comes from the natural isomorphism $\phi_{G}$ (cf. (13)) which induces a canonical isomorphism $P_{1}^{\overline{\mathcal{G}}}(G / N)=I(G) /\left(I\left(K G_{(2)}\right)+I^{2}(G)\right) \cong$ $G / K G_{(2)}$; it implies that $I(H) \cap M=I\left(H \cap K G_{(2)}\right)+I^{2}(H)$ since

$$
\begin{aligned}
\frac{I(H) \cap\left(I\left(K G_{(2)}\right)+I^{2}(G)\right)}{I^{2}(H)} & =\operatorname{Ker}\left(I(H) / I^{2}(H) \stackrel{\bar{i}}{\longrightarrow} I(G) / I\left(K G_{(2)}\right)+I^{2}(G)\right) \\
& =\phi_{H} \operatorname{Ker}\left(H / H_{2} \rightarrow G / K G_{(2)}\right) .
\end{aligned}
$$

Hence $I(H) / I(H) \cap M \cong H K G_{(2)} / K G_{(2)}$. Under these identifications the diagram in Corollary 2.3 (except from the Tor-terms) looks as follows where $J_{H}=J / I(H) J$, $T^{\prime}=\operatorname{Tor}_{1}^{\mathbb{Z}}\left(G / H K G_{(2)}, J_{H}\right)$ and where the top row is part of the corresponding 6term exact sequence with $\iota$ being induced by the inclusion $H \hookrightarrow G$ and $\pi$ being the canonical quotient map. Moreover, $\mu_{H}^{\prime}(\bar{h} \otimes \bar{x})=\overline{(h-1) x}$ and $\mu_{G}^{\prime}(\bar{g} \otimes \bar{x})=\overline{(g-1) x}$ for $h \in H, g \in G$ and $x \in J$.

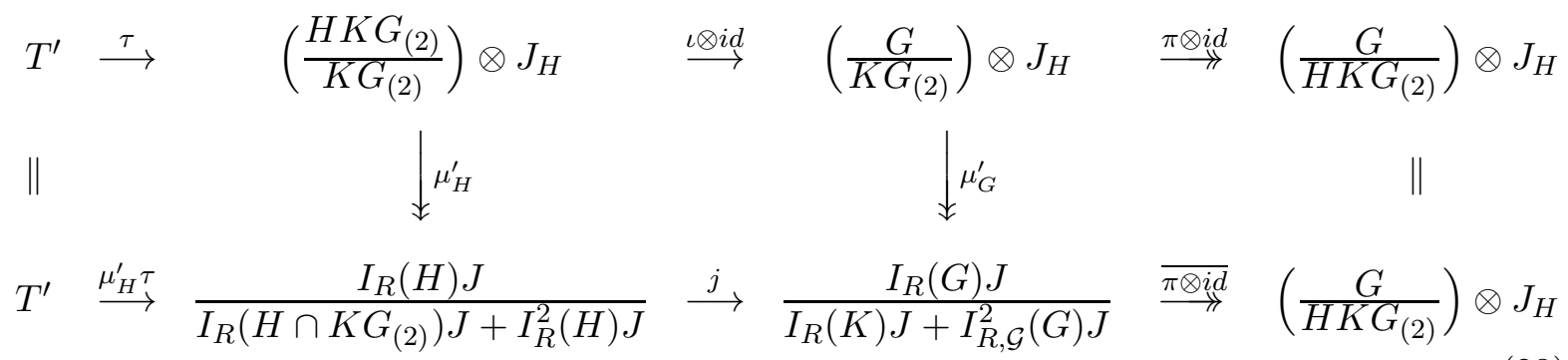

In view of the above remarks Corollary 2.3 amounts to the following functorial description of the $R$-module $I_{R}(G) J /\left(I_{R}(K) J+I_{R, \mathcal{G}}^{2}(G) J\right)$.

Theorem 3.3 The middle square of diagram (23) is a pushout of $R$-modules; in particular, $\operatorname{Ker}\left(\mu_{G}^{\prime}\right)=(\iota \otimes i d) \operatorname{Ker}\left(\mu_{H}^{\prime}\right)$. Moreover, the rows of the diagram above are exact; in particular, $\operatorname{Ker}(j)$ is generated by the cosets $\overline{(h-1) x}$ where $h \in H, x \in J$ such that there is some $k \in \mathbb{Z}$ for which $h \in K G_{(2)} G^{k}$ and 
$k x \in I(H) J$. Furthermore, omitting the terms $T^{\prime}$, the bottom sequence is induced by the top sequence via the map $\mu_{H}^{\prime}$.

The description of $\operatorname{Ker}(j)$ given in the theorem is obtained by using the canonical generators of the torsion product, see [19] V.6.

Corollary 3.4 If the sequence

$$
1 \rightarrow H K G_{(2)} / K G_{(2)} \rightarrow G / K G_{(2)} \rightarrow G / H K G_{(2)} \rightarrow 1
$$

of abelian groups splits then there is an isomorphism of $R$-modules

$$
\frac{I_{R}(G) J}{I_{R}(K) J+I_{R, \mathcal{G}}^{2}(G) J} \cong \frac{I_{R}(H) J}{I_{R}\left(H \cap K G_{(2)}\right) J+I_{R}^{2}(H) J} \oplus\left(\frac{G}{H K G_{(2)}}\right) \otimes\left(\frac{J}{I(H) J}\right) .
$$

In fact, here the top sequence in diagram (23) is split short exact whence so is the bottom sequence (omitting the terms $T^{\prime}$ ).

This last result in particular applies when $K$ is normal, $\mathcal{G}=\gamma$ and $H K / K$ is a semidirect factor of $G / K$ (normal or not). Thus we recover the corresponding results of Khambadkone and Karan-Vermani for $J=I(H)$ in [17, [15] and for $J=I^{m}(H)$ in [18], [14].

Another application of Theorem 3.3 is the following intersection theorem.

Corollary 3.5 For any subgroup $K$ of $G$ one has the identity

$$
I_{R}(H) J \cap\left(I_{R}(K) J+I_{R, \mathcal{G}}^{2}(G) J\right)=I_{R}\left(H \cap K G_{(2)}\right) J+I_{R}^{2}(H) J+U
$$

where the subgroup $U$ of $I_{R}(H) J$ is generated by the elements $(h-1) x$ where $h \in$ $H, x \in J$ for which there exists $k \in \mathbb{Z}$ such that $h \in K G_{(2)} G^{k}$ and $k x \in I(H) J$.

In particular, the groups $I^{n}(H) \cap\left(I(K) I^{n-1}(H)+I^{2}(G) I^{n-1}(H)\right)$ can be considered as being known for $n \leq 4$, using the computation of $I^{2}(H) / I^{3}(H)$ in [2] or [4] and of $I^{3}(H) / I^{4}(H)$ in [4].

In the case $R=\mathbb{Z}$ and $J=I(H)$ we can improve Theorem 3.3 , as follows. For a group $K$ with $\mathrm{N}$-series $\mathcal{K}$ there is a canonical homomorphism

$$
c_{2}^{K, \mathcal{K}}:\left(K / K_{(2)}\right) \wedge\left(K / K_{(2)}\right) \rightarrow K_{(2)} / K_{(3)}, \quad c_{2}^{K, \mathcal{K}}\left(\left(a K_{(2)}\right) \wedge\left(b K_{(2)}\right)\right)=[a, b] K_{(3)}
$$

for $a, b \in K$. We note $c_{2}^{G, \mathcal{G}}=c_{2}^{\mathcal{G}}$ and $c_{2}^{H, \gamma}=c_{2}^{H}$. Furthermore, for any abelian group there is a canonical homomorphism

$$
l_{2}(A): \quad A \wedge A \rightarrow A \otimes A, \quad l_{2}(A)(x \wedge y)=x \otimes y-y \otimes x
$$


for $x, y \in A$. We note $l_{2}\left(G / G_{(2)}\right)=l_{2}^{\mathcal{G}}, l_{2}\left(H / H_{2}\right)=l_{2}^{H}$ and $l_{2}^{\mathcal{G} H}=(\iota \otimes i d) l_{2}^{H}$ with $\iota: H / H_{2} \rightarrow G / K G_{(2)}$ induced by $H \hookrightarrow G$. Now consider the commutative square

$$
\begin{array}{ccc}
H^{a b} \wedge H^{a b} & \stackrel{l_{2}^{\mathcal{G} H}}{\longrightarrow} & \left(G / K G_{(2)}\right) \otimes\left(H / H_{2}\right) \\
\downarrow^{c_{2}^{H}} & & \mathfrak{l}^{\mu_{2}^{\mathcal{G} H}} \\
H_{2} / H_{3} & \stackrel{d_{2}^{\mathcal{G} H}}{\longrightarrow} & I(G) I(H) /\left(I(K) I(H)+I_{\mathcal{G}}^{2}(G) I(H)\right)
\end{array}
$$

where for $g \in G, h \in H$ and $x \in H_{2}$

$$
\mu_{2}^{\mathcal{G} H}\left(\left(g G_{(2)}\right) \otimes\left(h H_{2}\right)\right)=\overline{(g-1)(h-1)}, \quad d_{2}^{\mathcal{G} H}\left(x H_{3}\right)=\overline{x-1} .
$$

This diagram provides a simultaneous functorial description of both the second Fox quotient and the second Fox subgroup, in a completely symmetric way, as follows.

Theorem 3.6 Diagram (24) is a pushout square of abelian groups; in particular

$$
\begin{gathered}
I(G) I(H) /\left(I(K) I(H)+I_{\mathcal{G}}^{2}(G) I(H)\right) \cong\left(G / K G_{(2)}\right) \otimes\left(H / H_{2}\right) / l_{2}^{\mathcal{G} H} \operatorname{Ker}\left(c_{2}^{H}\right) \\
\left(G \cap\left(1+I(K) I(H)+I_{\mathcal{G}}^{2}(G) I(H)\right)\right) / H_{3}=\operatorname{Ker}\left(d_{2}^{\mathcal{G} H}\right)=c_{2}^{H} \operatorname{Ker}\left(l_{2}^{\mathcal{G} H}\right) .
\end{gathered}
$$

An analogous result holds for the third Fox quotient and subgroup, see Theorem 6.6 below.

Proof: In Proposition 2.2 take $n=2, J=I_{R}(H)$ and $U=I^{2}(H)$. Then using the isomorphisms $\phi_{H}$ and $\phi_{G}$ we see that the following diagram is a pushout square, with $\mathcal{H}=\gamma_{H}$.

$$
\begin{aligned}
& H^{a b} \otimes H^{a b} \otimes R \stackrel{\iota \otimes i d \otimes i d}{\longrightarrow} \quad\left(G / K G_{(2)}\right) \otimes H^{a b} \otimes R
\end{aligned}
$$

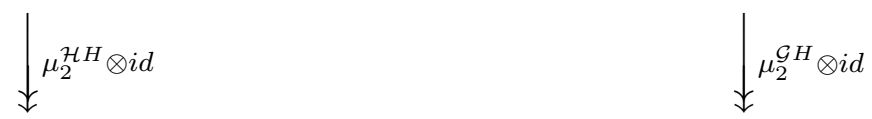

$$
\begin{aligned}
& I_{R}^{2}(H) / I_{R}^{3}(H) \quad \stackrel{j}{\longrightarrow} I_{R}(G) I_{R}(H) /\left(I_{R}(K) I_{R}(H)+I_{R, \mathcal{G}}^{2}(G) I_{R}(H)\right)
\end{aligned}
$$

So $\operatorname{Ker}\left(\mu_{2}^{\mathcal{G} H} \otimes i d\right)=(\iota \otimes i d \otimes i d) \operatorname{Ker}\left(\mu_{2}^{\mathcal{H} H} \otimes i d\right)$. But for $R=\mathbb{Z}, \operatorname{Ker}\left(\mu_{2}^{\mathcal{H} H}\right)=$ $l_{2}^{H} \operatorname{Ker}\left(c_{2}^{H}\right)$ by the isomorphism $I^{2}(H) / I^{3}(H) \cong \mathrm{U}_{2} \mathrm{~L}(H)$ obtained in [2]. Hence diagram (24) is a pushout, too (abstractly, this follows from the gluing lemma for pushouts in any category as the cited isomorphism means that diagram (24) for $K=\{1\}, G=H$ and $\mathcal{G}=\gamma$ is a pushout square). To deduce from this 
fact the second identity in the assertion, we first use Corollary 3.2 to observe that $G \cap(1+I(G) I(H))=H \cap\left(1+I(H)^{2}\right)=H_{2}$.

We remark that in [8] we deduce a more explicit description of the induced subgroup $G \cap\left(1+I_{R}(K) I_{R}(H)+I_{R}^{2}(G) I_{R}(H)\right)$ from the pushout square (25), for arbitrary subgroups $K, H$ of $G$ and coefficient rings $R$.

A nice generalization of Corollary 3.6 also holds for higher Fox quotients in the case where $H / H_{n}$ is free nilpotent which morally means that $H$ does not have any relations in lower commutator filtration. This is the matter of the next section.

\section{Fox quotients with respect to free nilpotent subgroups}

In Proposition 2.1 we saw that the map $\zeta_{n}^{\mathcal{G}}$ is an isomorphism if $H$ is free. In this section we compute its kernel for $\mathcal{G}=\gamma$ if only $H / H_{n}$ is free nilpotent of class $n-1$.

Let $\mathcal{L}\left(H^{a b}\right)$ and $T\left(H^{a b}\right)=\mathbb{Z} \oplus \bigoplus_{n>0}\left(H^{a b}\right)^{\otimes n}$ be the free Lie ring and the tensor ring over $H^{a b}$, respectively. Let $\mathrm{L}(H)$ be the graded Lie ring defined by the successive lower central quotients of $H$ and $\operatorname{Gr}(Z(H))$ be the associated graded ring of $Z Z(H)$ with respect to the filtration $I^{k}(H), k \geq 0$. Then we have natural homomorphisms of graded abelian groups (actually, of graded rings in the case of $q$ and of graded Lie rings in the case of $l$ and $c$ )

$$
\operatorname{Gr}(\mathbb{Z}(H)) \stackrel{\aleph^{q}}{\longleftarrow} T\left(H^{a b}\right) \stackrel{l}{\longleftarrow} \mathcal{L}\left(H^{a b}\right) \stackrel{c}{\longleftrightarrow} \mathrm{L}(H)
$$

provided by the identifications $q_{1}=l_{1}=c_{1}=i d_{H^{a b}}$ and the universal properties of $T\left(H^{a b}\right)$ and $\mathcal{L}\left(H^{a b}\right)$. If $H$ is free then $c$ is well-known to be an isomorphism. So for every $n \geq 2$ we obtain natural homomorphisms

$$
Q_{n-1}(G) \otimes H^{a b} \stackrel{Q_{n-1}}{\stackrel{(i) q_{n-1} \otimes i d}{\longleftarrow}}\left(H^{a b}\right)^{\otimes n} \stackrel{l_{n}}{\longleftarrow} \mathcal{L}_{n}\left(H^{a b}\right) \stackrel{c_{n}^{H}}{\longleftrightarrow} \mathrm{L}_{n}(H)
$$

The maps $l_{n}$ and $c_{n}$ may be viewed as sending a "formal" $n$-fold commutator in $\mathcal{L}_{n}\left(H^{a b}\right)$ to the corresponding tensor commutator and to the coset of the corresponding group commutator, respectively. With these notations, we get the following

Theorem 4.1 Let $n \geq 2$ and suppose that $H / H_{n}$ is a free nilpotent group of class $n-1$, i.e. $H / H_{n} \cong F / F_{n}$ for some free group $F$. Then the map $\zeta_{n}$ above induces a natural isomorphism

$$
I^{n-1}(G) I(H) / I^{n}(G) I(H) \cong Q_{n-1}(G) \otimes H^{a b} /\left(Q_{n-1}(i) q_{n-1} \otimes i d\right) l_{n} \operatorname{Ker}\left(c_{n}^{H}\right) .
$$

In particular, if $\mathrm{H} / \mathrm{H}_{n+1}$ is free nilpotent of class $n$, then

$$
I^{n-1}(G) I(H) / I^{n}(G) I(H) \cong Q_{n-1}(G) \otimes H^{a b} .
$$


We remark that the proof below shows that the group $\left(Q_{n-1}(i) q_{n-1} \otimes i d\right) l_{n} \operatorname{Ker}\left(c_{n}\right)$ is contained in the kernel of $\zeta_{n}$ for arbitary subgroups $H$; it actually equals $\operatorname{Ker}\left(\zeta_{n}\right)$ for $n=2$, by Theorem 3.6. In the remaining sections we study a refinement of the approximation of the $n$-th Fox quotient by $\zeta_{n}$ which takes this fact into account (among other phenomena).

Proof: We wish to apply Proposition 2.2 with $U=I^{n}(H)$. By assumption on

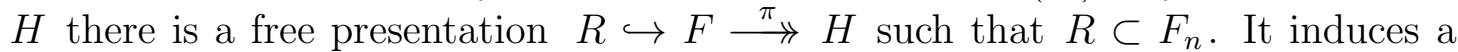
ring isomorphism $\mathbb{Z}(F) / I^{k}(F) \cong \mathbb{Z}(H) / I^{k}(H)$ for all $k \leq n$. Choose a basis $\left(f_{i}\right)_{i \in I}$ of $F$. Since $I(F)$ is a free $F$-module with basis $\left(f_{i}-1\right)_{i \in I}$, it follows that $I(H) / I^{n}(H)$ is a free $\mathbb{Z}(H) / I^{n-1}(H)$-module with basis $\left(\pi\left(f_{i}\right)-1\right)_{i \in I}$. Whence in diagram (18) (with $J=I(H))$ we obtain an isomorphism

$$
\xi: P_{n-1}(G) \otimes H^{a b} \stackrel{\cong}{\rightarrow} P_{n-1}(G) \otimes_{H} P_{n-1}(H)
$$

defined by $\xi\left(x \otimes \pi\left(f_{i}\right) H^{\prime}\right)=x \otimes\left(\pi\left(f_{i}\right)-1+I^{n}(H)\right)$. Since $H^{a b}$ is free abelian we get an isomorphism

$$
\xi(\iota \otimes i d): Q_{n-1}(G) \otimes H^{a b} \cong \operatorname{Im}\left(\left(Q_{n-1}(G) \otimes_{H} P_{n-1}(H)\right)\right.
$$

with the inclusion $\iota: Q_{n-1}(G) \hookrightarrow P_{n-1}(G)$. In order to compute $\operatorname{Ker}\left(\mu_{H}\right)$ in (18) write $T=T\left(H^{a b}\right)$ and let $\bar{T}$ be its augmentation ideal. It is well known that there is a ring isomorphism $\chi: T / \bar{T}^{k} \cong Z Z(F) / I^{k}(F)$ for all $k \geq 1$, defined by sending a generator $f_{i} F_{2}$ to $f_{i}-1+I^{k}(F)$. Thus we get a commutative square

$$
\begin{aligned}
& P_{n-1}(H) \otimes_{H} P_{n-1}(H) \underset{\chi \otimes \chi}{\stackrel{\cong}{\cong}}\left(\bar{T} / \bar{T}^{n}\right) \otimes_{T}\left(\bar{T} / \bar{T}^{n}\right) \quad \stackrel{\cong}{\stackrel{ }{\longrightarrow}} \quad \bar{T}^{2} / \bar{T}^{n+1} \\
& \downarrow \mu_{H} \\
& \frac{I^{2}(H)}{I^{n+1}(H)} \cong \frac{I^{2}(F)+I(R) Z Z(F)}{I^{n+1}(F)+I(R) Z Z(F)} \cong \bar{T}^{2} /\left(\bar{T}^{n+1}+p_{n} \operatorname{Ker}\left(\pi_{n}\right)\right)
\end{aligned}
$$

where $\nu$ is induced by multiplication in $T$ and where the maps $p_{n}, \pi_{n}$ are part of the following commutative diagram and are induced by sending $a \in F_{n}$ to $\chi^{-1}\left(a-1+I^{n+1}(F)\right)$ and to $\pi(a) H_{n+1}$, respectively. The isomorphisms in the bottom row above are then deduced from the relations $I(R) \subset I\left(F_{n}\right) \subset I^{n}(F)$.

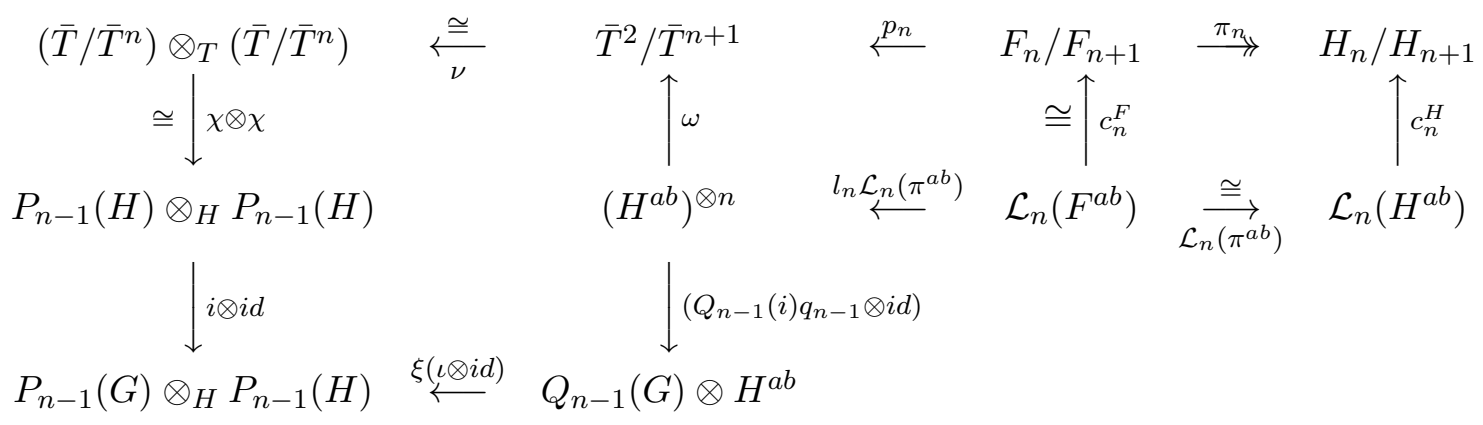


Here $\omega$ is the canonical injection. From the first of these two diagrams it follows that $\operatorname{Ker}\left(\mu_{H}\right)=(\chi \otimes \chi) \nu^{-1} p_{n} \operatorname{Ker}\left(\pi_{n}\right)$, so by 2.2 and the second diagram $\operatorname{Ker}\left(\mu_{G}\right)=(i \otimes i d)(\chi \otimes \chi) \nu^{-1} p_{n} \operatorname{Ker}\left(\pi_{n}\right)=(i \otimes i d)(\chi \otimes \chi) \nu^{-1} \omega l_{n} \operatorname{Ker}\left(c_{n}^{H}\right)=$ $\xi(\iota \otimes i d)\left(Q_{n-1}(i) q_{n-1} \otimes i d\right) l_{n} \operatorname{Ker}\left(c_{n}^{H}\right)$. By the equality $\mu_{G} \xi\left(\iota \otimes H^{a b}\right)=\zeta_{n}$ and by (27) this implies the assertion.

\section{Canonical approximation of Fox quotients}

In the preceding sections we computed the generalized Fox quotients $Q_{n}^{\mathcal{G}}(G, H)$ in some special cases by using the somewhat "naive" approximation $\zeta_{n}^{\mathcal{G}}$; we here study the deeper structure of the groups $Q_{n}^{\mathcal{G}}(G, H)$ by introducing a much closer approximation in terms of enveloping algebras which generalizes Quillen's approximation of augmentation quotients, i.e. the case $\mathcal{G}=\gamma$ and $H=G$. We start by recalling the latter construction.

The abelian group $\mathrm{L}^{\mathcal{G}}(G)=\sum_{n \geq 1} G_{(n)} / G_{(n+1)}$ is a graded Lie ring whose bracket is induced by the commutator pairing of $G$. So its enveloping algebra $\mathrm{UL}^{\mathcal{G}}(G)$ over the integers is defined. On the other hand, the filtration quotients $Q_{n}^{\mathcal{G}}(G)=I_{\mathcal{G}}^{n}(G) / I_{\mathcal{G}}^{n+1}(G)$ form the graded $\operatorname{ring} \operatorname{Gr}^{\mathcal{G}}(\mathbb{Z}(G))=\oplus_{n \geq 0} Q_{n}^{\mathcal{G}}(G)$; note that one has $\operatorname{Gr}(\mathbb{Z}(G)):=\operatorname{Gr}^{\gamma}(\mathbb{Z}(G))=\bigoplus_{n \geq 0} I^{n}(G) / I^{n+1}(G)$.

Now the map $\mathrm{L}^{\mathcal{G}}(G) \rightarrow \operatorname{Gr}^{\mathcal{G}}(\mathbb{Z}(G)), a G_{(n+1)} \mapsto a-1+I_{\mathcal{G}}^{n+1}(G)$ for $a \in G_{(n)}$, is a homomorphism of graded Lie rings and hence extends to a map of graded rings $\theta^{\mathcal{G}}: \mathrm{UL}^{\mathcal{G}}(G) \rightarrow \operatorname{Gr}^{\mathcal{G}}(\mathbb{Z}(G))$. This map is clearly surjective but rarely globally injective; for instance, $\theta^{\gamma}$ is injective if $G$ has torsionfree lower central quotients $G_{n} / G_{n+1}$ or is cyclic, but $\theta^{\gamma}$ is non injective for all non cyclic finite abelian groups [1. At least, the kernel of $\theta^{\mathcal{G}}$ is torsion as $\theta^{\mathcal{G}} \otimes \mathbb{Q}$ is an isomorphism; this was proved by Quillen for $\mathcal{G}=\gamma$ and follows from work of Hartley [11] in the general case, see also [9]. Moreover, $\operatorname{Ker}\left(\theta^{\mathcal{G}}\right)$ is trivial in degree 1 and 2 (by [2] for $N=\gamma$ ) and is explicitely known in degree 3, see [4].

To generalize the foregoing concept to Fox quotients consider the filtration

$$
\mathcal{F}^{1}=\mathbb{Z}(G) I(H) \supset \mathcal{F}^{2}=I(G) I(H) \supset \ldots \supset \mathcal{F}^{n}=I_{\mathcal{G}}^{n-1}(G) I(H) \supset \ldots
$$

of $\mathbb{Z}(G) I(H)$. The associated graded group $\operatorname{Gr}^{\mathcal{G} \gamma}(\mathbb{Z}(G) I(H))=\bigoplus_{n \geq 1} Q_{n}^{\mathcal{G}}(G, H)$ is a graded $\operatorname{Gr}^{\mathcal{G}}(Z Z(G))-\operatorname{Gr}(Z Z(H))$-bimodule in the canonical way, and hence a $\mathrm{UL}^{\mathcal{G}}(G)-\mathrm{UL}(H)$-bimodule via the maps $\theta^{\mathcal{G}}$ and $\theta^{\gamma_{H}}$. Now let $\mathcal{H}=\left(H_{(n)}\right)_{n \geq 1}$ be the N-series of $H$ defined by $H_{(n)}=H \cap G_{(n)}$. The injection $I(H) \hookrightarrow \mathbb{Z}(G) I(H)$ is a map of $\mathbb{Z}(H)$-bimodules taking $I^{n}(H)$ and $I_{\mathcal{H}}^{n-1}(H) I(H)$ into $\mathcal{F}^{n}$; it thus induces homomorphisms $\operatorname{Gr}(I(H)) \longrightarrow \operatorname{Gr}^{\mathcal{G} \gamma}(\mathbb{Z}(G) I(H)) \longleftarrow \operatorname{Gr}^{\mathcal{H} \gamma}(I(H))$ of graded $\operatorname{Gr}(\mathbb{Z}(H))$-bimodules and $\operatorname{Gr}^{\mathcal{H}}(\mathbb{Z}(H))-\operatorname{Gr}(\mathbb{Z}(H))$-bimodules, resp. So by extension of scalars along the graded ring homomorphisms $\operatorname{Gr}(\mathbb{Z}(H)) \rightarrow$ $\operatorname{Gr}^{\mathcal{G}}(\mathbb{Z}(G))$ and $\operatorname{Gr}^{\mathcal{H}}(\mathbb{Z}(H)) \rightarrow \mathrm{Gr}^{\mathcal{G}}(\mathbb{Z}(G))$ induced by the injection $H \hookrightarrow G$ we get natural surjective maps of graded $\operatorname{Gr}^{\mathcal{G}}(Z Z(G))-\operatorname{Gr}(Z Z(H))$-bimodules

$$
\xi_{\mathcal{G} \gamma}^{G H}: \operatorname{Gr}^{\mathcal{G}}(\mathbb{Z}(G)) \otimes_{\operatorname{Gr}(\boldsymbol{Z}(H))} \operatorname{Gr}(I(H)) \longrightarrow \operatorname{Gr}^{\mathcal{G} \gamma}(\mathbb{Z}(G) I(H)),
$$




$$
\xi_{\mathcal{G H}}^{G H}: \operatorname{Gr}^{\mathcal{G}}(\mathbb{Z}(G)) \otimes_{\operatorname{Gr}^{\mathcal{H}}(\boldsymbol{Z}(H))} \operatorname{Gr}^{\mathcal{H} \gamma}(I(H)) \longrightarrow \operatorname{Gr}^{\mathcal{G} \gamma}(\mathbb{Z}(G) I(H)) .
$$

It is convenient to combine $\xi_{\mathcal{G} \gamma}^{G H}$ with Quillen's approximation to obtain an epimorphism of graded $\mathrm{UL}^{\mathcal{G}}(G)-\mathrm{UL}(H)$-bimodules

$$
\theta^{\mathcal{G} H}=\xi_{\mathcal{G} \gamma}^{G H}\left(\theta^{\mathcal{G}} \otimes \theta^{\gamma_{H}}\right): \quad \mathrm{U}^{\mathcal{G}}(G, H):=\mathrm{UL}^{\mathcal{G}}(G) \otimes_{\mathrm{UL}(H)} \overline{\mathrm{UL}}(H) \longrightarrow \operatorname{Gr}^{\mathcal{G} \gamma}(Z Z Z(G) I(H))
$$

where $\overline{\mathrm{UL}}(H)$ denotes the augmentation ideal of $\mathrm{UL}(H)$.

Remark 5.1 The approximation of $\operatorname{Gr}^{\mathcal{G} \gamma}(Z Z Z(G) I(H))$ by $\xi_{\mathcal{G} \mathcal{H}}^{G H}$ is a priori "closer" than the one by $\xi_{\mathcal{G} \gamma}^{G H}$ as is seen from the following commutative diagram

$$
\begin{aligned}
& \operatorname{Gr}^{\mathcal{G}}(\mathbb{Z}(G)) \otimes_{\operatorname{Gr}(\boldsymbol{Z}(H))} \operatorname{Gr}(I(H)) \stackrel{\cong}{\stackrel{\cong}{m}} \operatorname{Gr}^{\mathcal{G}}(\mathbb{Z}(G)) \otimes_{\operatorname{Gr}^{\mathcal{H}}(\boldsymbol{Z}(H))}\left(\operatorname{Gr}^{\mathcal{H}}(\mathbb{Z}(H)) \otimes_{\operatorname{Gr}(\boldsymbol{Z}(H))} \operatorname{Gr}(I(H))\right) \\
& \downarrow_{\xi_{\mathcal{G} \gamma}^{G H}} \downarrow \downarrow i d \otimes \xi_{\mathcal{H} \gamma}^{H H} \\
& \operatorname{Gr}^{\mathcal{G} \gamma}(\mathbb{Z}(G) I(H)) \quad \stackrel{\xi_{\mathcal{G H}}^{G H}}{\leftrightarrows} \quad \operatorname{Gr}^{\mathcal{G}}(\mathbb{Z}(G)) \otimes_{\operatorname{Gr}^{\mathcal{H}}(\boldsymbol{Z}(H))} \operatorname{Gr}^{\mathcal{H} \gamma}(I(H))
\end{aligned}
$$

where $m$ is the canonical isomorphism. But as our goal is to approximate the group $\operatorname{Gr}^{\mathcal{G} \gamma}(\mathbb{Z}(G) I(H))$ in terms of enveloping algebras we do not to care about this difference.

For $i \geq 0$ and $j \geq 1$ let

$$
\nu_{i j}: \mathrm{U}_{i} \mathrm{~L}^{\mathcal{G}}(G) \otimes \mathrm{U}_{j} \mathrm{~L}(H) \rightarrow \mathrm{U}_{i+j}^{\mathcal{G}}(G, H)
$$

be the canonical map. Note that for any group $K$ the ring $\operatorname{UL}(K)$ is generated by $\mathrm{U}_{1} \mathrm{~L}(K)=\mathrm{L}_{1}(K) \cong K^{a b}$. This implies that $\nu_{(n-1) 1}$ is surjective, and also implies exactness of the following sequence of graded $\mathrm{UL}^{\mathcal{G}}(G)-\mathrm{UL}(H)$-bimodules

$$
\mathrm{UL}^{\mathcal{G}}(G) \otimes \mathrm{U}_{1} \mathrm{~L}(H) \otimes \overline{\mathrm{UL}}(H) \stackrel{\psi}{\longrightarrow} \mathrm{UL}^{\mathcal{G}}(G) \otimes \overline{\mathrm{UL}}(H) \stackrel{q}{\longrightarrow} \mathrm{U}^{\mathcal{G}}(G, H) \rightarrow 0
$$

where $\psi(x \otimes y \otimes z)=x y \otimes z-x \otimes y z$. It is now easy to compute $\mathrm{U}_{n}^{\mathcal{G}}(G, H)$ for $n \leq 3$, also using the identity $\operatorname{Ker}\left(\mu_{2}^{H}\right)=l_{2}^{H} \operatorname{Ker}\left(c_{2}^{H}\right)$, cf. the proof of Theorem 3.6.

From now on we abbreviate $G^{A B}=G / G_{(2)}$.

Proposition 5.2 There are canonical isomorphisms

$$
\begin{gathered}
\mathrm{U}_{1}^{\mathcal{G}}(G, H) \cong \mathrm{U}_{1} \mathrm{~L}(H) \cong H^{a b} \\
\mathrm{U}_{2}^{\mathcal{G}}(G, H) \cong \frac{G^{A B} \otimes H^{a b}}{l_{2}^{\mathcal{G} H} \operatorname{Ker}\left(c_{2}^{H}\right)}
\end{gathered}
$$




$$
\begin{aligned}
& \mathrm{U}_{3}^{\mathcal{G}}(G, H) \cong \operatorname{coker}\left(\epsilon=\left(\begin{array}{ccc}
c_{2}^{\mathcal{G}} \otimes i d & 0 & 0 \\
-l_{2}^{\mathcal{G}} \otimes i d & i^{\mathcal{G} H H} & i^{H H H}
\end{array}\right)\right) \\
& \left(\left(G^{A B} \wedge G^{A B}\right) \otimes H^{a b}\right) \quad \oplus \quad\left(G^{A B} \otimes l_{2}^{H} \operatorname{Ker}\left(c_{2}^{H}\right)\right) \quad \oplus \quad l_{33}^{H} \operatorname{Ker}\left(c_{33}^{H}\right) \\
& \downarrow \epsilon \\
& \left(\left(G_{(2)} / G_{(3)}\right) \otimes H^{a b}\right) \quad \oplus \quad\left(G^{A B} \otimes G^{A B} \otimes H^{a b}\right)
\end{aligned}
$$

where the homomorphisms $\mathrm{L}_{3}(H) \stackrel{c_{33}^{H}}{\longleftarrow}\left(H^{a b}\right)^{\otimes 3} \stackrel{l_{33}^{H}}{\longrightarrow}\left(H^{a b}\right)^{\otimes 3}$ are defined such that for $x, y, z \in H^{a b}, c_{33}^{H}(x \otimes y \otimes z)$ is the triple Lie bracket $[x,[y, z]]$ in the Lie algebra $\mathrm{L}(H)$ and $l_{33}^{H}(x \otimes y \otimes z)$ is the triple Lie bracket $[x,[y, z]]$ in the tensor algebra $T\left(H^{a b}\right)$. Furthermore, we note $i^{\mathcal{G H H}}=i d \otimes \iota \otimes i d: G^{A B} \otimes H^{a b} \otimes H^{a b} \rightarrow$ $G^{A B} \otimes G^{A B} \otimes H^{a b}$ and $i^{H H H}=\iota \otimes \iota \otimes i d: H^{a b} \otimes H^{a b} \otimes H^{a b} \rightarrow G^{A B} \otimes G^{A B} \otimes H^{a b}$.

As an immediate consequence of this computation and of Proposition 3.1 and Theorem 3.6 we get

Proposition 5.3 For all groups $G$ and subgroups $H$ of $G$ the maps $\xi_{n}^{\mathcal{G H}}$ and $\theta_{n}^{\mathcal{G} H}$ are isomorphisms for $n=1,2$.

One may also ask when $\theta^{\mathcal{G H}}$ is globally an isomorphism. We have a positive answer in at least one case.

Corollary 5.4 If $\mathcal{G}$ has torsionfree factors and $H$ is a free group then $\theta^{\mathcal{G} H}$ is an isomorphism.

Proof: We have the following commutative diagram

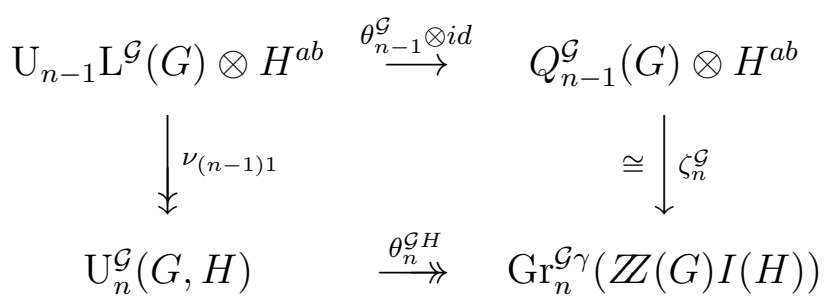

where $\zeta_{n}^{\mathcal{G}}$ is an isomorphism by Proposition [2.1. Moreover, $\theta^{\mathcal{G}}$ here is an isomorphism since $\mathcal{G}$ has torsionfree factors [9], whence $\nu_{(n-1) 1}$ and $\theta_{n}^{\mathcal{G H}}$ are isomorphisms, too.

We now exhibit a canonical part of the kernel of $\theta^{\mathcal{G} H}$ (other than $\operatorname{Ker}\left(\theta^{\mathcal{G}} \otimes \theta^{\gamma_{H}}\right)$ ) in comparing $\mathcal{G}$ to the lower central series of $H$, as follows. 
For elements $x_{1}, \ldots, x_{m}$ of any ring define the iterated commutator $\left[x_{1}, \ldots, x_{m}\right]$ to be $x_{1}$ if $m=1$ and to be $\left[x_{1},\left[x_{2}, \ldots,\left[x_{m-1}, x_{m}\right] \ldots\right]\right.$ if $m \geq 2$. In the latter case one has the formula

$$
\left[x_{1}, \ldots, x_{m}\right]=\sum_{s=0}^{m-1}(-1)^{m-s+1} \sum_{J} x_{i_{1}} \cdots x_{i_{s}} x_{m} x_{j_{1}} \cdots x_{j_{m-1-s}}
$$

where $J=\left\{\left(i_{1}, \ldots, i_{s}, j_{1}, \ldots, j_{m-1-s}\right) \| 1 \leq i_{1}<\ldots<i_{s} \leq m-1 \geq j_{1}>\ldots>\right.$ $j_{m-1-s} \geq 1$ such that $\left.\left\{i_{1}, \ldots, i_{s}, j_{1}, \ldots, j_{m-1-s}\right\}=\{1, \ldots, m-1\}\right\}$. We say that an $m$-tuple $\underline{h}=\left(h_{1}, \ldots, h_{m}\right)$ of elements of $H$ is of height $\geq n$ if $h_{j} \in H_{k_{j}} \cap G_{\left(l_{j}\right)}$ such that $l_{1}+\ldots+l_{m}-l_{j}+k_{j}=n$ for $1 \leq j \leq m$. For such an $m$-tuple $\underline{h}$ let $r_{1}(\underline{h})=h_{1}$ if $m=1$ and if $m \geq 2$,

$$
\begin{gathered}
r_{1}(\underline{h})=\left[h_{1},\left[h_{2}, \ldots,\left[h_{m-1}, h_{m}\right] \ldots\right] \in H\right. \\
r_{2}(\underline{h})=\sum_{s=0}^{m-1}(-1)^{m-s+1} \sum_{J}\left(h_{i_{1}} G_{\left(l_{i_{1}}+1\right)}\right) \ldots\left(h_{i_{s}} G_{\left(l_{i_{s}}+1\right)}\right) .\left(h_{m} G_{\left(l_{m}+1\right)}\right) .\left(h_{j_{1}} G_{\left(l_{j_{1}}+1\right)}\right) \ldots \\
\left(h_{j_{m-2-s}} G_{\left(l_{j_{m-2-s}}+1\right)}\right) \otimes\left(h_{j_{m-1-s}} H_{k_{j_{m-1}-s}+1}\right) \in\left(\mathrm{UL}^{\mathcal{G}}(G) \otimes \mathrm{UL}(H) \overline{\mathrm{UL}}(H)\right)_{n}
\end{gathered}
$$

Here, and throughout the rest of this paper, we consider the cosets $h_{j} H_{k_{j}+1} \in$ $\mathrm{L}_{k_{j}}(H)$ and $h_{j} G_{\left(l_{j}+1\right)} \in \mathrm{L}_{l_{j}}^{\mathcal{G}}(G)$ as elements of $\mathrm{U}_{k_{j}} \mathrm{~L}(H)$ and $\mathrm{U}_{l_{j}} \mathrm{~L}^{\mathcal{G}}(G)$, resp., suppressing the canonical map $\mathrm{L}^{\mathcal{K}}(K) \rightarrow \mathrm{UL}^{\mathcal{K}}(K)$ from the notation. Moreover, all products denoted by . are taken in the respective enveloping algebras.

For $n \geq 2$ let $\mathcal{R}_{n}^{\mathcal{G} H}$ be the subgroup of $\mathrm{UL}^{\mathcal{G}}(G) \otimes_{\mathrm{UL}(H)} \overline{\mathrm{UL}}(H)$ generated by the elements

$$
R_{n}\left(\underline{h}_{1}, \ldots, \underline{h}_{p}\right)=1_{\mathrm{UL}^{\mathcal{G}}(G)} \otimes\left(r_{1}\left(\underline{h}_{1}\right) \cdots r_{1}\left(\underline{h}_{p}\right) H_{n+1}\right)-\sum_{q=1}^{p} r_{2}\left(\underline{h}_{q}\right) \in \mathrm{U}_{n}^{\mathcal{G}}(G, H)
$$

where $p \geq 1$ and each $\underline{h}_{q}$ is an $m_{q}$-tuple, $m_{q} \geq 2$, of height $\geq n$ such that $r_{1}\left(\underline{h}_{1}\right) \cdots r_{1}\left(\underline{h}_{p}\right) \in H_{n}$. The term $\mathcal{R}_{3}^{\mathcal{G} H}$ will be considered in Corollary 6.7 below.

Proposition 5.5 One has identities $\theta^{\mathcal{G} H}\left(\mathcal{R}_{n}^{\mathcal{G} H}\right)=0$ and $\mathcal{R}_{n}^{\mathcal{G} H} \overline{\mathrm{UL}}(H)=0$. Moreover, in the definition of $\mathcal{R}_{n}^{\mathcal{G} H}$ it suffices to take only those tupels $\underline{h}_{q}=$ $\left(h_{q 1}, \ldots, h_{q m_{q}}\right)$ for which $l_{q j} \geq k_{q j}$ for $1 \leq j \leq m_{q}$.

Proof: Let $\underline{h}=\left(h_{1}, \ldots, h_{m}\right) \in H^{m}$ be of height $\geq n$. We contend that in $\mathbb{Z}(G), r_{1}(\underline{h})-1 \in \mathcal{F}^{n}$ and if $m \geq 2$,

$$
r_{1}(\underline{h})-1 \equiv\left[h_{1}-1,\left[\ldots,\left[h_{m-1}-1, h_{m}-1\right] \ldots\right] \bmod \mathcal{F}^{n+1} .\right.
$$


We proceed by induction on $m$. The case $m=1$ being obvious let $m \geq 2$. Note that $\underline{h}^{\prime}=\left(h_{2}, \ldots, h_{m}\right)$ is of height $\geq n-l_{1}$; hence by induction hypothesis, $r_{1}\left(\underline{h}^{\prime}\right)-1 \in \mathcal{F}^{n-l_{1}}$ and $x=r_{1}\left(\underline{h}^{\prime}\right)-1-\left[h_{2}-1,\left[\ldots,\left[h_{m-1}-1, h_{m}-1\right] \ldots\right] \in\right.$ $\mathcal{F}^{n-l_{1}+1}$. On the other hand, $r_{1}\left(\underline{h}^{\prime}\right)-1 \in \theta^{\mathcal{G}}\left(\mathcal{G}_{l_{2}+\ldots+l_{m}}\right) \subset I_{\mathcal{G}}^{l_{2}+\ldots+l_{m}}(G)$ and that $x \in I_{\mathcal{G}}^{l_{2}+\ldots+l_{m}+1}(G)$ since $\theta^{\mathcal{G}}$ is a graded Lie map. Now

$$
r_{1}(\underline{h})-1=\left(\left(h_{1}-1\right)\left(r_{1}\left(\underline{h}^{\prime}\right)-1\right)-\left(r_{1}\left(\underline{h}^{\prime}\right)-1\right)\left(h_{1}-1\right)\right) h_{1}^{-1} r_{1}\left(\underline{h}^{\prime}\right)^{-1} .
$$

By the above remarks, $\left(h_{1}-1\right)\left(r_{1}\left(\underline{h}^{\prime}\right)-1\right)-\left(r_{1}\left(\underline{h}^{\prime}\right)-1\right)\left(h_{1}-1\right) \in I_{\mathcal{G}}^{l_{1}}(G) \mathcal{F}^{n-l_{1}}+$ $I_{\mathcal{G}}^{l_{2}+\ldots+l_{m}}(G) I^{k_{1}}(H) \subset \mathcal{F}^{n}$ by the immediate relation $I_{\mathcal{G}}^{l}(G) \mathcal{F}^{e} I^{k}(H) \subset \mathcal{F}^{l+e+k}$ for $l, e, k \geq 0$. Thus also $r_{1}(\underline{h})-1 \in \mathcal{F}^{n}$. Writing $h_{1}^{-1} r_{1}\left(\underline{h}^{\prime}\right)^{-1}=1+\left(h_{1}^{-1} r_{1}\left(\underline{h}^{\prime}\right)^{-1}-1\right)$ we get

$$
\begin{aligned}
r_{1}(\underline{h})-1 & \equiv\left(h_{1}-1\right)\left(r_{1}\left(\underline{h}^{\prime}\right)-1\right)-\left(r_{1}\left(\underline{h}^{\prime}\right)-1\right)\left(h_{1}-1\right) \bmod \mathcal{F}^{n+1} \\
& =\left[h_{1}-1, x+\left[h_{2}-1,\left[\ldots,\left[h_{m-1}-1, h_{m}-1\right] \ldots\right]\right]\right. \\
& \equiv\left[h_{1}-1,\left[h_{2}-1,\left[\ldots,\left[h_{m-1}-1, h_{m}-1\right] \ldots\right]\right] \bmod \mathcal{F}^{n+1}\right.
\end{aligned}
$$

since $\left(h_{1}-1\right) x-x\left(h_{1}-1\right) \in I_{\mathcal{G}}^{l_{1}}(G) \mathcal{F}^{n-l_{1}+1}+I_{\mathcal{G}}^{l_{2}+\ldots+l_{m}+1}(G) I^{k_{1}}(H) \subset \mathcal{F}^{n+1}$. Hence (29) is proved. Using the identity $a b-1=(a-1)+(b-1)+(a-1)(b-1)$ we obtain

$$
\begin{aligned}
\theta^{\mathcal{G H}}\left(1_{\mathrm{UL}}(G) \otimes r_{1}\left(\underline{h}_{1}\right) \ldots r_{1}\left(\underline{h}_{p}\right) H_{n+1}\right) & =r_{1}\left(\underline{h}_{1}\right) \ldots r_{1}\left(\underline{h}_{p}\right)-1+\mathcal{F}^{n+1} \\
& =\sum_{q=1}^{p} r_{1}\left(\underline{h}_{q}\right)-1+\mathcal{F}^{n+1} \\
& =\sum_{q=1}^{p} \theta^{\mathcal{G} H}\left(r_{2}\left(\underline{h}_{q}\right)\right)+\mathcal{F}^{n+1}
\end{aligned}
$$

where the last identity is due to relations (29) and (28). Hence $\theta^{\mathcal{G} H}\left(\mathcal{R}_{n}^{\mathcal{G} H}\right)=0$. Next we prove the last part of the assertion. First note that for $\underline{h}$ as above

$$
l_{1}+\ldots+l_{m}=n+l_{j}-k_{j}
$$

for $1 \leq j \leq m$. Now suppose that for some $q, l_{q 1}+\ldots+l_{q m_{q}}<n$ which by (30) means that $l_{q j}<k_{q j}$ for all $j$ from 1 to $m_{q}$. For clarity we suppress the index $q$ from the notation. We have $h_{j} \in H_{k_{j}} \subset G_{\left(k_{j}\right)} \subset G_{\left(l_{j}+1\right)}$, so $r_{2}\left(\underline{h}_{q}\right)=0$ since all terms to the left of $\otimes$ are trivial. On the other hand,

$$
\begin{aligned}
k_{1}+\ldots+k_{m} & =n-\left(l_{2}+\ldots+l_{m}\right)+k_{2}+\ldots+k_{m} \\
& =n+\left(k_{2}-l_{2}\right)+\ldots+\left(k_{m}-l_{m}\right) \\
& \geq n+m-1 \\
& \geq n+1
\end{aligned}
$$


so $r_{1}\left(\underline{h}_{q}\right) \in H_{k_{1}+\ldots+k_{m}} \subset H_{n+1}$. Hence $R_{n}\left(\underline{h}_{1}, \ldots, \underline{h}_{p}\right)=R_{n}\left(\underline{h}_{1}, \ldots, \underline{\hat{h}}_{q}, \ldots, \underline{h}_{p}\right)$ where $\left(\underline{h}_{1}, \ldots, \underline{\hat{h}}_{q}, \ldots, \underline{h}_{p}\right)$ is $\left(\underline{h}_{1}, \ldots, \underline{h}_{p}\right)$ with $\underline{h}_{q}$ omitted. Thus we may suppose that in the definition of $\mathcal{R}_{n}^{\mathcal{G H} H}$, for all $q$ from 1 to $p$, one has $l_{q 1}+\ldots+l_{q m_{q}} \geq n$, i.e., $l_{q j} \geq k_{q j}$ for $1 \leq j \leq m_{q}$ by (30). Under this hypothesis let $v \in \overline{\mathrm{UL}}(H)$ and let us show that $R_{n}\left(\underline{h}_{1}, \ldots, \underline{h}_{p}\right) \cdot v=0$. We have

$$
R_{n}\left(\underline{h}_{1}, \ldots, \underline{h}_{p}\right) \cdot v=\left(r_{1}\left(\underline{h}_{1}\right) \cdots r_{1}\left(\underline{h}_{p}\right) G_{(n+1)}\right) \otimes v-\sum_{q=1}^{p} r_{2}^{G}\left(\underline{h}_{q}\right) \otimes v
$$

where

$$
\begin{gathered}
r_{2}^{G}(\underline{h})=\sum_{s=0}^{m-1}(-1)^{m-s+1} \sum_{J}\left(h_{i_{1}} G_{\left(l_{i_{1}}+1\right)}\right) \ldots\left(h_{i_{s}} G_{\left(l_{i s}+1\right)}\right) \cdot\left(h_{m} G_{\left(l_{m}+1\right)}\right) \cdot\left(h_{j_{1}} G_{\left(l_{j_{1}}+1\right)}\right) \ldots \\
\left(h_{j_{m-2-s}} G_{\left(l_{j_{m-2-s}}+1\right)}\right) \cdot\left(h_{j_{m-1-s}} G_{\left(k_{j_{m-1-s}}+1\right)}\right)
\end{gathered}
$$

Suppose that for some $q, l_{q 1}+\ldots+l_{q m_{q}}>n$, i.e., $l_{q j}>k_{q j}$ for $1 \leq j \leq m_{q}$. Then $h_{q j} G_{\left(k_{q j}+1\right)}=0$ as $h_{q j} \in G_{\left(l_{q j}\right)} \subset G_{\left(k_{q j}+1\right)}$, so $r_{2}^{G}\left(\underline{h}_{q}\right)=0$ since all the last factors in the sum defining it are trivial. On the other hand, $r_{1}\left(\underline{h}_{q}\right) \in$ $G_{\left(l_{q 1}+\ldots+l_{\left.q m_{q}\right)}\right)} \subset G_{(n+1)}$, so $R_{n}\left(\underline{h}_{1}, \ldots, \underline{h}_{p}\right) \cdot v=R_{n}\left(\underline{h}_{1}, \ldots, \underline{\hat{h}}_{q}, \ldots, \underline{h}_{p}\right) \cdot v$. Thus we can finally assume that for all $q$ from 1 to $p, l_{q 1}+\ldots+l_{q m_{q}}=n$, i.e., $l_{q j}=k_{q j}$ for $1 \leq j \leq m_{q}$ by (30). Here $r_{2}^{G}\left(\underline{h}_{q}\right)=\left[h_{q 1} G_{\left(l_{q 1}+1\right)}, \ldots, h_{q m_{q}} G_{\left(l_{q m_{q}}+1\right)}\right]=$ $\left[h_{q 1},\left[\ldots,\left[h_{q\left(m_{q}-1\right)}, h_{q m_{q}}\right] \ldots\right] G_{\left(l_{q 1}+\ldots+l_{q m_{q}}+1\right)}=r_{1}\left(\underline{h}_{q}\right) G_{(n+1)}\right.$ by definition of the Lie ring $\mathrm{L}^{\mathcal{G}}(G)$. On the other hand, $r_{1}\left(\underline{h}_{1}\right) \cdots r_{1}\left(\underline{h}_{p}\right) G_{(n+1)}=\sum_{q=1}^{p} r_{1}\left(\underline{h}_{q}\right) G_{(n+1)}$ since $r_{1}\left(\underline{h}_{q}\right) \in G_{\left(l_{q 1}+\ldots+l_{q m_{q}}\right)}=G_{(n)}$ for each $q$. So $R_{n}\left(\underline{h}_{1}, \ldots, \underline{h}_{p}\right) \cdot v=0$, as desired.

Proposition 5.5 implies that the quotient group

$$
\overline{\mathrm{U}}^{\mathcal{G}}(G, H) \stackrel{\text { def }}{=} \mathrm{U}^{\mathcal{G}}(G, H) / \sum_{n \geq 2} \mathrm{UL}^{\mathcal{G}}(G) \mathcal{R}_{n}^{\mathcal{G} H}
$$

is a graded $\mathrm{UL}^{\mathcal{G}}(G)-\mathrm{UL}(H)$-bimodule, and that $\theta^{\mathcal{G} H}$ induces a surjective homomorphism of graded $\mathrm{UL}^{\mathcal{G}}(G)-\mathrm{UL}(H)$-bimodules

$$
\bar{\theta}^{\mathcal{G} H}: \overline{\mathrm{U}}^{\mathcal{G}}(G, H) \rightarrow \mathrm{Gr}^{\mathcal{G} \gamma}(\mathbb{Z}(G) I(H)) .
$$

Note that $\overline{\mathrm{U}}^{\gamma}(G, G)=\mathrm{U}^{\gamma}(G, G) \cong \overline{\mathrm{UL}}(G)$ and that $\bar{\theta}^{\gamma G}=\theta^{\gamma G}$ coincides in positive degrees with the map $\theta^{\gamma}$ constructed by Quillen. By analogy with the fact that $\theta^{\mathcal{G}} \otimes \mathbb{Q}$ is always an isomorphism we pose the following

Problem 5.6 Is it true that the epimorphism

$$
\bar{\theta}_{n}^{\mathcal{G} H} \otimes \mathbb{Q}: \overline{\mathrm{U}}_{n}^{\mathcal{G}}(G, H) \otimes \mathbb{Q} \rightarrow Q_{n}^{\mathcal{G}}(G, H) \otimes \mathbb{Q}
$$

is an isomorphism for all groups $G$, subgroups $H$ and $n \geq 1$ ? In other words, is $\operatorname{Ker}\left(\theta^{\mathcal{G} H}\right)$ a torsion group? 
The answer is affirmative in degree $n \leq 2$ by Proposition 5.3 and also in degree 3 by Corollary 6.2 below.

\section{The third Fox quotient}

In this section all proofs are postponed to the end.

The structure of $Q_{3}^{\mathcal{G}}(G, H)$ is completely determined by Proposition 5.2 and the following result.

Theorem 6.1 For all groups $G$ and subgroups $H$ there is a natural exact sequence

$\operatorname{Tor}_{1}^{\mathbb{Z}}\left(G^{A B}, H^{a b}\right) \oplus\left(\operatorname{Ker}\left(l_{2}^{\mathcal{G} H}\right) \cap \operatorname{Ker}\left(c_{2}^{H}\right)\right) \stackrel{\left(\delta_{1}, \delta_{2}\right)}{\longrightarrow} \mathrm{U}_{3}^{\mathcal{G}}(G, H) \stackrel{\theta_{3}^{\mathcal{G} H}}{\longrightarrow} Q_{3}^{\mathcal{G}}(G, H) \rightarrow 0$

Here $\delta_{1}$ is a homomorphism while $\delta_{2}$ is a secondary operator which is a welldefined homomorphism only modulo $\operatorname{Im}\left(\delta_{1}\right)$, i.e. an additive relation with indeterminacy $\operatorname{Im}\left(\delta_{1}\right)$. The construction of $\delta_{1}$ and $\delta_{2}$ is given in (36) and (53) below in a functorial manner and in (37) and (38) in an explicit form.

Corollary 6.2 Problem 5.6 has an affirmative answer for $n=3$.

Let us discuss Theorem 6.1 in a number of special cases. First suppose that $H=G$.

If $\mathcal{G}=\gamma$ the map $l_{2}^{\mathcal{G} G}$ is injective; hence the theorem formally generalizes the description of $\operatorname{Ker}\left(\theta_{3}^{\mathcal{G}}\right)$ in [4] (in the case $\mathcal{G}=\gamma$ ). If $\mathcal{G} \neq \gamma$ the result is still of interest as groups of the type $Q_{n}^{\mathcal{G}}(G, G)$ occur in the study of Fox quotients of semidirect products, see [16]. In particular, if $G$ is the semidirect product of a normal subgroup $H$ and a subgroup $K$ the quotient

$$
X=\frac{I^{3}(H) \oplus I([H, K]) I(H)}{I^{4}(H)+I(H) I([H, K]) I(H)+I([H, K, H]) I(H)+I([H, K, K]) I(H)}
$$

is proved to be a direct summand of $Q_{3}(G, H)$ but is not computed in [16]; we here fill this gap noting that $X=Q_{3}^{\mathcal{H}}(H, H)$ where the N-series $\mathcal{H}=\left(H_{(n)}\right)_{n \geq 1}$ is given by $H_{(n+1)}=\left[H_{(n)}, G\right]$, see also [9]. Indeed, the structure of $Q_{3}^{\mathcal{G}}(G, G)$ is determined by the following two corollaries, first from a functorial point of view further developed in remark 6.4, then by means of an explicit formula. 
Corollary 6.3 The group $Q_{3}^{\mathcal{G}}(G, G)$ is determined by the following tower of successive natural quotients

$$
\begin{array}{ccc}
\operatorname{Tor}_{1}^{\mathbb{Z}}\left(G^{A B}, G^{a b}\right) \stackrel{\delta_{1}}{\longrightarrow} & \mathrm{U}_{3}^{\mathcal{G}}(G, G) \\
\operatorname{Ker}\left(\tilde{c}_{2}^{G}:\left(G_{(2)} / G_{2}\right) \wedge\left(G_{(2)} / G_{2}\right) \rightarrow G_{2} / G_{3}\right) & \stackrel{\delta_{21} \rightarrow}{\longrightarrow} \operatorname{Coker}\left(\delta_{1}\right) \\
\operatorname{Ker}\left([,] \tau_{1}: \operatorname{Tor}_{1}^{\mathbb{Z}}\left(G^{A B}, G^{A B}\right) \rightarrow G_{2} /\left[G_{(2)}, G_{(2)}\right] G_{3}\right) \stackrel{q_{21}}{\longrightarrow} & \operatorname{Coker}\left(\delta_{21}\right) \\
& \downarrow q_{22} \\
& \operatorname{Coker}\left(\delta_{22}\right) \\
& \cong \tilde{\theta}_{3}^{\mathcal{G} G} \\
& Q_{3}^{\mathcal{G}}(G, G)
\end{array}
$$

where $\tilde{c}_{2}^{G}$ is given by restriction of $c_{2}^{G}, \tilde{\theta}_{3}^{\mathcal{G} G}$ is induced by $\theta_{3}^{\mathcal{G} G}, \tau_{1}$ appears in the following part of a 6-term-exact sequence

$\operatorname{Tor}_{1}^{\mathbb{Z}}\left(G^{A B}, G^{A B}\right) \stackrel{\tau_{1}}{\longrightarrow} G^{A B} \otimes\left(G_{(2)} / G_{2}\right) \stackrel{i d \otimes i}{\longrightarrow} G^{A B} \otimes G^{a b} \stackrel{i d \otimes \pi}{\longrightarrow} G^{A B} \otimes G^{A B} \rightarrow 0$, $[]:, G^{A B} \otimes\left(G_{(2)} / G_{2}\right) \rightarrow G_{2} /\left[G_{(2)}, G_{(2)}\right] G_{3}$ is induced by the commutator pairing of $G$, and $\delta_{21}, \delta_{22}$ are induced by $\delta_{2}$, cf. the proof at the end of the section.

It actually follows from Lemma 6.7 below that $\operatorname{Im}\left(\delta_{21}\right)=q_{1}\left(\mathcal{R}_{3}^{\mathcal{G} G}\right)$. So if we replace $\mathrm{U}_{3}^{\mathcal{G}}(G, G)$ by $\overline{\mathrm{U}}_{3}^{\mathcal{G}}(G, G)$ the above tower reduces to just the two steps involving $\delta_{1}$ and $\delta_{22}$.

Remark 6.4 A similar description can be given in the general case $(H \neq G)$ by adding one additional step at the bottom of the tower. Indeed, there is an isomorphism $\tilde{\theta}_{3}^{\mathcal{G} H}: \operatorname{Coker}\left(\delta_{23}\right) \rightarrow Q_{3}^{\mathcal{G}}(G, H)$ where the construction of $\delta_{21}, \delta_{22}$ resembles the one in Corollary 6.3 and where $\delta_{23}$ looks at follows:

$$
\begin{aligned}
\operatorname{Ker}\left(\operatorname { K e r } \left(\operatorname{Tor}_{1}^{\mathbb{Z}}\left(G / H G_{(2)}, H^{a b}\right) \stackrel{\sigma_{1}}{\longrightarrow}\right.\right. & \left.\left.\operatorname{SP}^{2}\left(H G_{(2)} / G_{(2)}\right)\right) \stackrel{\sigma_{2}}{\longrightarrow} \operatorname{Coker}\left([,] \tau_{1}\right)\right) \\
& \downarrow \delta_{23}
\end{aligned}
$$

$\operatorname{Coker}\left(\delta_{22}\right)$ 
for suitable natural maps $\sigma_{1}, \sigma_{2}, \delta_{23}$ and where $\mathrm{SP}^{2}$ denotes the second symmetric tensor power. We renounce to give the precise definitions and the proof as this description may be of no practical use, but we mention it in order to illustrate our guiding philosophy: any natural construction of an abelian group associated with a nilpotent group (all kinds of augmentation and dimension quotients, homology etc.) should be functorially expressable in terms of (generally higher order) operators between suitable polynomial endofunctors of $\mathbf{A b}$ and their derived functors, applied to appropriate abelian subquotients of the nilpotent group in question (here $\mathbf{A b}$ denotes the category of abelian groups). For more examples of this structural phenomenon see also [4], [6], [8], [9], [10].

Corollary 6.5 There is a natural isomorphism $Q_{3}^{\mathcal{G}}(G, G) \cong \mathrm{U}_{3}^{\mathcal{G}}(G, G) /\left(U_{1}+U_{2}\right)$ induced by $\theta_{3}^{\mathcal{G} G}$ where

- $U_{1}$ is the subgroup of $\mathrm{U}_{3}^{\mathcal{G}}(G, G)$ generated by the elements

$\left(a G_{(2)}\right) \otimes\left(b^{k} H_{3}\right)-\left(a^{k} G_{(3)}\right) \otimes\left(b G_{2}\right)+\left(\begin{array}{l}k \\ 2\end{array}\right)\left(\left(a G_{(2)}\right)^{2} \otimes\left(b G_{2}\right)-\left(a G_{(2)}\right) \otimes\left(b G_{2}\right)^{2}\right)$

where $a, b \in G, k \in \mathbb{Z}$ such that $a^{k} \in G_{(2)}$ and $b^{k} \in G_{2}$, and as usual, $\left(a G_{(2)}\right)^{2}=$ $\left(a G_{(2)}\right) \cdot\left(a G_{(2)}\right)$, same for $\left(b G_{(2)}\right)^{2}$;

- $U_{2}$ is the set of elements

$$
\begin{gathered}
\sum_{q=1}^{p}\left(a_{q} G_{(3)}\right) \otimes\left(b_{q} G_{2}\right)-\left(b_{q} G_{(3)}\right) \otimes\left(a_{q} G_{2}\right)+\sum_{r=1}^{s}\left(c_{r}^{k_{r}} G_{(3)}\right) \otimes\left(d_{r} G_{2}\right)-\left(d_{r}^{k_{r}} G_{(3)}\right) \otimes\left(c_{r} G_{2}\right) \\
\quad+\sum_{r=1}^{s}\left(\begin{array}{c}
k_{r} \\
2
\end{array}\right)\left(\left(c_{r} G_{(2)}\right) \cdot\left(\left(d_{r} G_{(2)}\right)-\left(c_{r} G_{(2)}\right)\right) \otimes\left(d_{r} G_{2}\right)\right)-1 \otimes\left(g G_{4}\right)
\end{gathered}
$$

where $a_{q}, b_{q} \in G_{(2)}, c_{r}, d_{r} \in G, k_{r} \in \mathbb{Z}$ such that $c_{r}^{k_{r}}, d_{r}^{k_{r}} \in G_{(2)}$ for $1 \leq r \leq s$ and $g=\prod_{q=1}^{p}\left[a_{q}, b_{q}\right] \prod_{r=1}^{s}\left[c_{r}, d_{r}^{k_{r}}\right] \in G_{3}$.

The proof below shows that $U_{1}+U_{2}$ is indeed a subgroup of $\mathrm{U}_{3}^{\mathcal{G}}(G, G)$.

Construction of $\delta_{1}$. For a group $K$ and N-series $\mathcal{K}$ of $K$ the isomorphisms $\theta_{n}^{K, \mathcal{K}}: \mathrm{U}_{n} \mathrm{~L}^{\mathcal{K}}(K) \stackrel{\cong}{\longrightarrow} Q_{2}^{\mathcal{K}}(K), n=1,2$, provide natural exact sequences

$$
\begin{gathered}
0 \rightarrow \mathrm{U}_{2} \mathrm{~L}^{\mathcal{G}}(G) \stackrel{\mu_{2}^{\mathcal{G}}}{\longrightarrow} P_{2}^{\mathcal{G}}(G) \stackrel{\rho^{\mathcal{G}}}{\longrightarrow} G^{A B} \rightarrow 0 \\
0 \rightarrow \mathrm{U}_{2} \mathrm{~L}(H) \stackrel{\mu_{2}^{H}}{\longrightarrow} P_{2}(H) \stackrel{\rho^{H}}{\longrightarrow} H^{a b} \rightarrow 0
\end{gathered}
$$


Tensoring sequence (32) by $H^{a b}=\mathrm{U}_{1} \mathrm{~L}(H)$ and sequence (33) by $G^{A B}=\mathrm{U}_{1} \mathrm{~L}^{\mathcal{G}}(G)$ gives rise to natural exact sequences

$$
\begin{aligned}
& \operatorname{Tor}_{1}^{\mathbb{Z}}\left(G^{A B}, H^{a b}\right) \stackrel{\tau_{G}}{\longrightarrow} \mathrm{U}_{2} \mathrm{~L}^{\mathcal{G}}(G) \otimes \mathrm{U}_{1} \mathrm{~L}(H) \stackrel{\mu_{2}^{\mathcal{G} \otimes i d}}{\longrightarrow} P_{2}^{\mathcal{G}}(G) \otimes H^{a b} \stackrel{\rho^{\mathcal{G}} \otimes i d}{\longrightarrow} G^{A B} \otimes H^{a b} \rightarrow 0 \\
& \operatorname{Tor}_{1}^{\mathbb{Z}}\left(G^{A B}, H^{a b}\right) \stackrel{\tau_{H}}{\longrightarrow} \mathrm{U}_{1} \mathrm{~L}^{\mathcal{G}}(G) \otimes \mathrm{U}_{2} \mathrm{~L}(H) \stackrel{i d \otimes \mu_{2}^{H}}{\longrightarrow} G^{A B} \otimes P_{2}(H) \stackrel{i d \otimes \rho^{H}}{\longrightarrow} G^{A B} \otimes H^{a b} \rightarrow 0
\end{aligned}
$$

Then define

$$
\delta_{1}=\nu_{12} \tau_{H}-\nu_{21} \tau_{G}: \operatorname{Tor}_{1}^{\mathbb{Z}}\left(G^{A B}, H^{a b}\right) \quad \longrightarrow \quad \mathrm{U}_{3}^{\mathcal{G}}(G, H) .
$$

Note that $\delta_{1}$ essentially is the difference between a left and a right connecting homomorphism, kind of asymmetry phenomenon which also induces the non-trivial torsion relations in the non-abelian tensor square and the second homology of 2 step nilpotent groups, cf. [6].

To describe $\delta_{1}$ more explicitly let $\langle\bar{g}, k, \bar{h}\rangle$ be a typical generator of $\operatorname{Tor}_{1}^{\mathbb{Z}}\left(G^{A B}, H^{a b}\right)$, i.e. a symbol where $k \in \mathbb{Z}, \bar{g}=g G_{(2)} \in \mathrm{U}_{1} \mathrm{~L}^{\mathcal{G}}(G), \bar{h}=h H_{2} \in \mathrm{U}_{1} \mathrm{~L}(H)$ for $g \in G$, $h \in H$ such that $g^{k} \in G_{(2)}$ and $h^{k} \in H_{2}$, cf. [19] V.6. Then

$$
\delta_{1}\langle\bar{g}, k, \bar{h}\rangle=\left(\bar{g} \otimes\left(h^{k} H_{3}\right)-\left(g^{k} G_{(3)}\right) \otimes \bar{h}+\left(\begin{array}{l}
k \\
2
\end{array}\right)\left(\bar{g}^{2} \otimes \bar{h}-\bar{g} \otimes \bar{h}^{2}\right) .\right.
$$

where $\bar{g}^{2}=\bar{g} \cdot \bar{g}$ and $\bar{h}^{2}=\bar{h} \cdot \bar{h}$.

Explicit formula for $\delta_{2}$ (the functorial construction is given in (153) below). Suppose that $H^{a b}$ is finitely generated (the general case can be deduced from Lemmas 3.5 and 3.6 in [8] exactly in the same way as what follows). Then there exists a decomposition $H^{a b}=\bigoplus_{k=1}^{r} \mathbb{Z} / d_{k} \mathbb{Z} \cdot\left(h_{k} H_{2}\right)$ with $h_{k} \in H, d_{k} \in \mathbb{N}$. Let $x \in H^{a b} \wedge H^{a b}$. Then $x=\sum_{1 \leq i<j \leq r} a_{i j}\left(h_{i} H_{2}\right) \wedge\left(h_{j} H_{2}\right)$ with $a_{i j} \in \mathbb{Z}$. By Lemmas 3.5 and 3.6 in [8] one has $x \in \operatorname{Ker}\left(l_{2}^{G H}\right)$ if and only if for all $1 \leq k \leq r$, $\prod_{1 \leq i<k} h_{i}^{a_{i k}} \prod_{k<j \leq n} h_{j}^{-a_{k j}} \in G_{(2)} G^{d_{k}}$, with $G^{d}=\left\{g^{d} \mid g \in G\right\}$. Now suppose that $x \in \operatorname{Ker}\left(l_{2}^{G H}\right) \cap \operatorname{Ker}\left(c_{2}^{H}\right)$. Then $\gamma:=\prod_{1 \leq i<j \leq r}\left[h_{i}, h_{j}\right]^{a_{i j}} \in H_{3}$, and for all $1 \leq k \leq r$ there are $g_{k}^{\prime} \in G_{(2)}$ and $g_{k} \in G$ such that $\prod_{l=1}^{r} h_{l}^{\alpha_{k l}}=g_{k}^{\prime} g_{k}^{d_{k}}$ where $\alpha_{k l}=a_{l k}$ if $l<k, \alpha_{k l}=0$ if $l=k$, and $\alpha_{k l}=-a_{k l}$ if $l>k$. Then

$$
\begin{aligned}
\delta_{2}(x)= & \sum_{k=1}^{r}\left(\left(g_{k}^{\prime} G_{(3)}\right) \otimes\left(h_{k} H_{2}\right)+\left(g_{k} G_{(2)}\right) \otimes\left(h_{k}^{d_{k}} H_{3}\right)+\left(\begin{array}{c}
d_{k} \\
2
\end{array}\right)\left(g_{k} G_{(2)}\right) \cdot\left(\left(g_{k} G_{(2)}\right)\right.\right. \\
& \left.-\left(h_{k} G_{(2)}\right)\right) \otimes\left(h_{k} H_{2}\right)-1 \otimes\left(\left(\gamma H_{4}\right)+\sum_{l=1}^{r}\left(\begin{array}{c}
\alpha_{k l} \\
2
\end{array}\right)\left(h_{l} H_{2}\right)^{2} \cdot\left(h_{k} H_{2}\right)\right. \\
& \left.\left.+\sum_{1 \leq p<q \leq r} \alpha_{k p} \alpha_{k q}\left(h_{p} H_{2}\right) \cdot\left(h_{q} H_{2}\right) \cdot\left(h_{k} H_{2}\right)\right)\right)+\operatorname{Im}\left(\delta_{1}\right)
\end{aligned}
$$


with $\left(h_{l} H_{2}\right)^{2}=\left(h_{l} H_{2}\right) \cdot\left(h_{l} H_{2}\right)$.

The starting point of the proof of Theorem 6.1 is the following description of the third relative Fox polynomial group which is completely analogous to our description of the second one in Theorem 3.6 .

Let $N$ be a normal subgroup of $G$ and consider the following diagram.

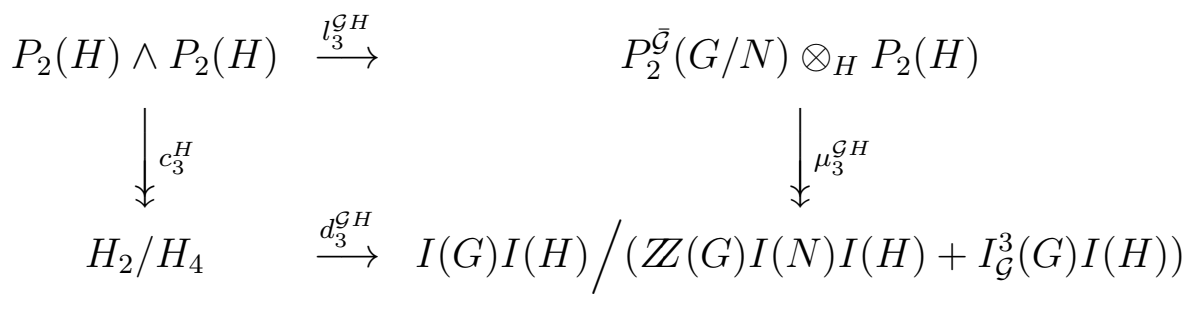

where for $a, b, h \in H, g \in G$ and $x \in H_{2}$

$l_{3}^{\mathcal{G} H}\left(p_{2}(a) \wedge p_{2}(b)\right)=p_{2}(a) \otimes p_{2}(b)-p_{2}(b) \otimes p_{2}(a)-\left[p_{2}(a), p_{2}(b)\right] \otimes\left(p_{2}(a)+p_{2}(b)\right)$,

$c_{3}^{H}\left(\left(a H_{2}\right) \wedge\left(b H_{2}\right)\right)=[a, b] H_{4}, d_{3}^{\mathcal{G} H}\left(x H_{4}\right)=x-1+\mathbb{Z}(G) I(N) I(H)+I_{\mathcal{G}}^{3}(G) I(H)$, and $\mu_{3}^{\mathcal{G} H}\left(\left(p_{2}(g) \otimes\left(p_{2}(h)\right)\right)=(g-1)(h-1)+Z Z(G) I(N) I(H)+I_{\mathcal{G}}^{3}(G) I(H)\right.$.

This diagram provides a simultaneous functorial description of both the third relative Fox polynomial group and the third relative Fox subgroup, in exactly the same way as the second Fox quotient and the second Fox subgroup are determined in Theorem 3.6, as follows.

Theorem 6.6 Diagram (39) is a pushout square of abelian groups; in particular

$$
\begin{gathered}
I(G) I(H) /\left(\mathbb{Z}(G) I(N) I(H)+I_{\mathcal{G}}^{3}(G) I(H)\right) \cong\left(P_{2}^{\overline{\mathcal{G}}}(G / N) \otimes_{H} P_{2}(H)\right) / l_{3}^{\mathcal{G} H}\left(\operatorname{Ker}\left(c_{3}^{H}\right)\right) \\
\left(G \cap\left(1+\mathbb{Z}(G) I(N) I(H)+I_{\mathcal{G}}^{3}(G) I(H)\right)\right) / H_{4}=\operatorname{Ker}\left(d_{3}^{\mathcal{G} H}\right)=c_{3}^{H}\left(\operatorname{Ker}\left(l_{3}^{\mathcal{G} H}\right)\right) .
\end{gathered}
$$

The second equality should allow to explicitly compute the third relative Fox subgroup, in a similar way as we deduce in [8] the second relative Fox-subgroup from Theorem 3.6 .

Proof: Same principle as in the proof of 3.6: diagram (39) is a pushout as it is obtained by gluing the following two pushouts where $\mathcal{H}=\gamma_{H}$ :

$$
\begin{array}{cccc}
P_{2}(H) \wedge P_{2}(H) & \stackrel{l_{3}^{\mathcal{H} H}}{\longrightarrow} & P_{2}(H) \otimes_{H} P_{2}(H) \stackrel{i \otimes i d}{\longrightarrow} & P_{2}^{\overline{\mathcal{G}}}(G / N) \otimes_{H} P_{2}(H) \\
\downarrow_{c_{3}^{H}} & \downarrow^{\mu_{3}^{\mathcal{H} H}=\mu_{H}} & \downarrow^{\mu_{3}^{\mathcal{G} H}=\mu_{G}} \\
H_{2} / H_{4} & \stackrel{d_{3}^{\mathcal{H} H}}{\longrightarrow} & I^{2}(H) / I^{4}(H) \stackrel{j}{\longrightarrow} & \frac{I(G) I(H)}{\mathbb{Z}(G) I(N) I(H)+I_{\mathcal{G}}^{3}(G) I(H)}
\end{array}
$$


In fact, the left hand square is a pushout by Theorem 3.5 in [4] and the right hand square by Proposition 2.3.

Proof of Theorem 6.1. First note that by (321), (33) homomorphisms

$$
\mathrm{U}_{2} \mathrm{~L}^{\mathcal{G}}(G) \otimes H^{a b} \stackrel{\alpha_{G}}{\longrightarrow} \frac{P_{2}^{\mathcal{G}}(G) \otimes P_{2}(H)}{\operatorname{Im}\left(Q_{2}^{\mathcal{G}}(G) \otimes P_{2}(H)^{2}\right)} \stackrel{\alpha_{H}}{\longleftarrow} G^{A B} \otimes \mathrm{U}_{2} \mathrm{~L}(H)
$$

are welldefined as being factorizations of the maps

$$
\mathrm{U}_{2} \mathrm{~L}^{\mathcal{G}}(G) \otimes P_{2}(H) \stackrel{q\left(\mu_{2}^{\mathcal{G} \otimes i d}\right)}{\longrightarrow} \frac{P_{2}^{\mathcal{G}}(G) \otimes P_{2}(H)}{\operatorname{Im}\left(Q_{2}^{\mathcal{G}}(G) \otimes P_{2}(H)^{2}\right)} \stackrel{q\left(i d \otimes \mu_{2}^{H}\right)}{\longleftarrow} P_{2}^{\mathcal{G}}(G) \otimes \mathrm{U}_{2} \mathrm{~L}(H)
$$

through $i d \otimes \rho^{H}$ and $\rho^{\mathcal{G}} \otimes i d$, resp., where $q: P_{2}^{\mathcal{G}}(G) \otimes P_{2}(H) \rightarrow \frac{P_{2}^{\mathcal{G}}(G) \otimes P_{2}(H)}{\operatorname{Im}\left(Q_{2}^{\mathcal{G}}(G) \otimes P_{2}(H)^{2}\right)}$ is the canonical projection. Consider the following commutative diagram where $\beta_{G}=q_{\otimes} \alpha_{G}$ with $q_{\otimes}: \frac{P_{2}^{\mathcal{G}}(G) \otimes P_{2}(H)}{\operatorname{Im}\left(Q_{2}^{\mathcal{G}}(G) \otimes P_{2}(H)^{2}\right)} \rightarrow P_{2}^{\mathcal{G}}(G) \otimes_{H} P_{2}(H)$ being the canonical surjection.

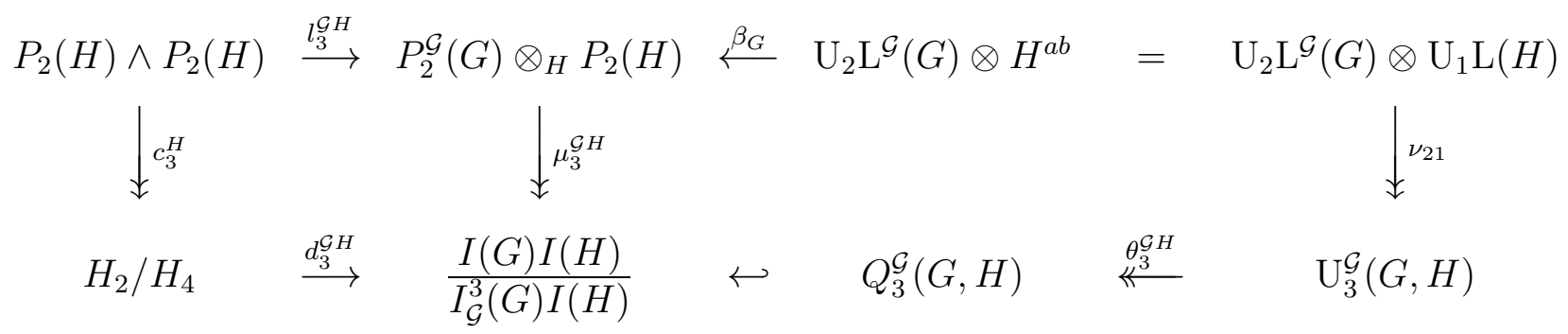

As $\operatorname{Ker}\left(\mu_{3}^{\mathcal{G} H}\right)=l_{3}^{\mathcal{G} H} \operatorname{Ker}\left(c_{3}^{H}\right)$ by Theorem 6.6 we have

$$
\operatorname{Ker}\left(\theta_{3}^{\mathcal{G} H}\right)=\nu_{21} \beta_{G}^{-1} l_{3}^{\mathcal{G} H} \operatorname{Ker}\left(c_{3}^{H}\right) .
$$

Thus the proof naturally divides in three steps:

Step 1: proving the identity

$$
\nu_{21} \operatorname{Ker}\left(\beta_{G}\right)=\operatorname{Im}\left(\delta_{1}\right) ;
$$

Step 2: giving a natural construction of $\delta_{2}$ and showing that

$$
\nu_{21} \beta_{G}^{-1} l_{3}^{\mathcal{G} H} \operatorname{Ker}\left(c_{3}^{H}\right) \equiv \operatorname{Im}\left(\delta_{2}\right) \bmod \operatorname{Im}\left(\delta_{1}\right) .
$$

Step 3: verifying the explicit formula for $\delta_{2}$ given by (38).

Step 1. Let $m_{2}^{\mathcal{G}}: G^{A B} \otimes G^{A B} \rightarrow \mathrm{U}_{2} \mathrm{~L}^{\mathcal{G}}(G)$ be the map given by multiplication in the ring $\mathrm{U}_{2} \mathrm{~L}^{\mathcal{G}}(G)$, and let $m_{2}^{H}=m_{2}^{\gamma_{H}}$. By definition of the tensor product over $H$ there is an exact sequence

$$
G^{A B} \otimes H^{a b} \otimes H^{a b} \stackrel{\psi}{\longrightarrow} \frac{P_{2}^{\mathcal{G}}(G) \otimes P_{2}(H)}{\operatorname{Im}\left(Q_{2}^{\mathcal{G}}(G) \otimes P_{2}(H)^{2}\right)} \stackrel{q_{\otimes}}{\longrightarrow} P_{2}^{\mathcal{G}}(G) \otimes_{H} P_{2}(H) \rightarrow 0
$$


with $\psi=\alpha_{G}\left(m_{2}^{\mathcal{G}} \otimes i d\right) i^{\mathcal{G} H H}-\alpha_{H}\left(i d \otimes m_{2}^{H}\right)$. Consider the following commutative diagram with exact rows and columns by (32), (33).

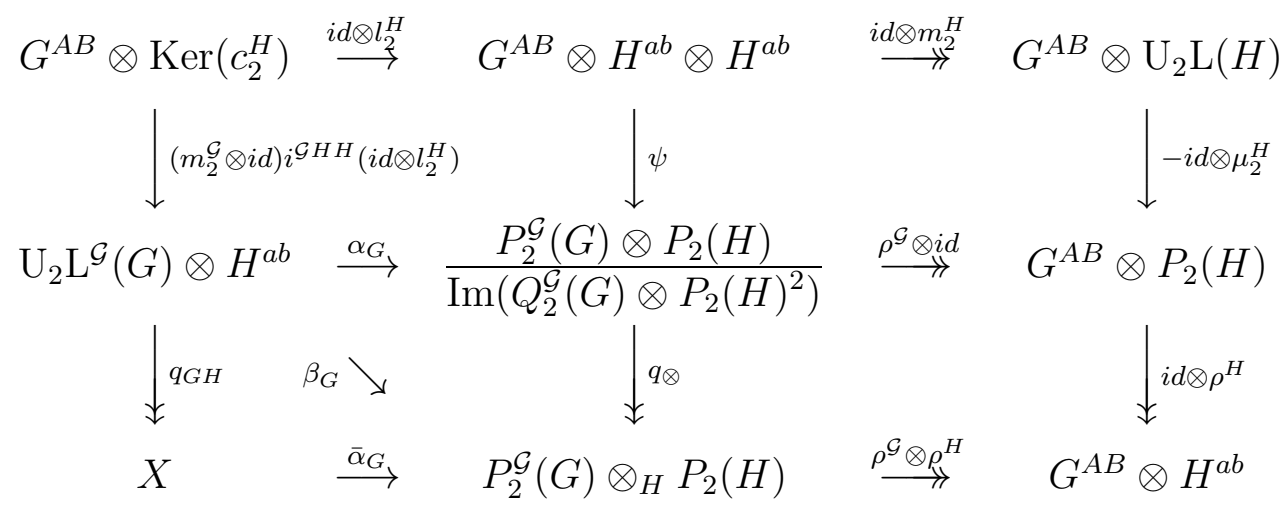

where

$$
X=\operatorname{Coker}\left(\left(m_{2}^{\mathcal{G}} \otimes i d\right) i^{\mathcal{G} H H}\left(i d \otimes l_{2}^{H}\right)\right)
$$

and where $q_{G H}$ is the canonical projection. Then by the snake lemma there is a canonical connecting homomorphism

$$
\omega: \operatorname{Ker}\left(i d \otimes \mu_{2}^{H}\right)=\operatorname{Im}\left(\tau_{H}\right) \rightarrow X / q_{G H} \operatorname{Ker}\left(\alpha_{G}\right),
$$

$\omega=q_{X} q_{G H} \alpha_{G}^{-1} \psi\left(i d \otimes m_{2}^{H}\right)^{-1}$ with $q_{X}: X \rightarrow X / q_{G H} \operatorname{Ker}\left(\alpha_{G}\right)$, satisfying the relation

$$
q_{X} \operatorname{Ker}\left(\bar{\alpha}_{G}\right)=\operatorname{Im}(\omega) .
$$

In order to compute $\operatorname{Im}(\omega)$ we consider the two summands of $\psi$ separately. As to the first one, we have

$$
q_{X} q_{G H} \alpha_{G}^{-1} \alpha_{G}\left(m_{2}^{\mathcal{G}} \otimes i d\right) i^{\mathcal{G} H H}\left(i d \otimes m_{2}^{H}\right)^{-1} \tau_{H}=q_{X} \bar{i}^{\overline{\mathcal{G H H}}} \tau_{H}
$$

where $\overline{i^{\mathcal{G H H}}}$ fits into the commutative diagram

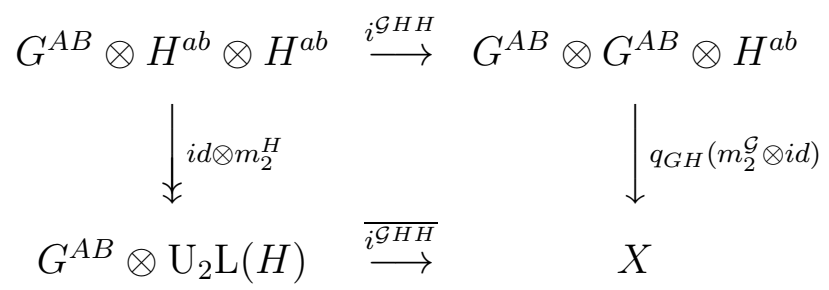

As to the second summand of $\psi$, let $\left\langle g G_{(2)}, k, h H_{2}\right\rangle$ be a typical generator of $\operatorname{Tor}_{1}^{\mathbb{Z}}\left(G^{A B}, H^{a b}\right)$ with $g \in G, h \in H, k \in \mathbb{Z}$ such that $g^{k} \in G_{(2)}$ and $h^{k} \in H_{2}$. 
Then

$$
\begin{aligned}
\alpha_{H}\left(i d \otimes m_{2}^{H}\right)\left(i d \otimes m_{2}^{H}\right)^{-1} \tau_{H}\left\langle g G_{(2)}, k, h H_{2}\right\rangle & =\alpha_{H}\left(\left(g G_{(2)}\right) \otimes\left(\mu_{2}^{H}\right)^{-1}\left(k p_{2}(h)\right)\right) \\
& =q\left(i d \otimes \mu_{2}^{H}\right)\left(p_{2}(g) \otimes\left(\mu_{2}^{H}\right)^{-1}\left(k p_{2}(h)\right)\right) \\
& =q\left(p_{2}(g) \otimes k p_{2}(h)\right) \\
& =q\left(k p_{2}(g) \otimes p_{2}(h)\right) \\
& =q\left(\mu_{2}^{\mathcal{G}} \otimes i d\right)\left(\left(\mu_{2}^{\mathcal{G}}\right)^{-1}\left(k p_{2}(g)\right) \otimes p_{2}(h)\right) \\
& =\alpha_{G}\left(\left(\mu_{2}^{\mathcal{G}}\right)^{-1}\left(k p_{2}(g)\right) \otimes\left(h H_{2}\right)\right) \\
& =\alpha_{G} \tau_{G}\left\langle g G_{(2)}, k, h H_{2}\right\rangle
\end{aligned}
$$

From (46) and (47) we obtain the relations

$$
\omega \tau_{H}=q_{X}\left(\overline{i^{\mathcal{G H H}}} \tau_{H}-q_{G H} \tau_{G}\right)
$$

whence by (45)

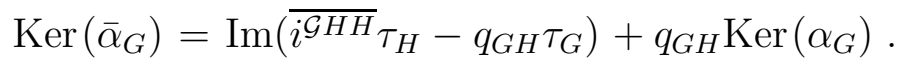

Next we compute $\operatorname{Ker}\left(\alpha_{G}\right)$. Consider the following commutative diagram whose top row is part of the six-term exact sequence obtained by tensoring sequence (32) with $P_{2}(H)$.

$$
\begin{aligned}
& \operatorname{Tor}_{1}^{\mathbb{Z}}\left(G^{A B}, P_{2}(H)\right) \stackrel{\tau}{\longrightarrow} \mathrm{U}_{2} \mathrm{~L}^{\mathcal{G}}(G) \otimes P_{2}(H) \stackrel{\mu_{2}^{\mathcal{G}} \otimes i d}{\longrightarrow} \quad P_{2}^{\mathcal{G}}(G) \otimes P_{2}(H) \\
& \downarrow \operatorname{Tor}_{1}^{\boldsymbol{Z}}\left(i d, \rho^{H}\right) \downarrow i d \otimes \rho^{H} \downarrow q \\
& \operatorname{Tor}_{1}^{\mathbb{Z}}\left(G^{A B}, H^{a b}\right) \quad \stackrel{\tau_{G}}{\longrightarrow} \quad \mathrm{U}_{2} \mathrm{~L}^{\mathcal{G}}(G) \otimes H^{a b} \quad \stackrel{\alpha_{G}}{\longrightarrow} \quad \frac{P_{2}^{\mathcal{G}}(G) \otimes P_{2}(H)}{\operatorname{Im}\left(Q_{2}^{\mathcal{G}}(G) \otimes P_{2}(H)^{2}\right)}
\end{aligned}
$$

The left hand square commutes by naturality of the connecting homomorphism and the right hand square by definition of $\alpha_{G}$. As $\operatorname{Ker}(q)=\left(\mu_{2}^{\mathcal{G}} \otimes i d\right) \operatorname{Ker}\left(i d \otimes \rho^{H}\right)$ we have

$$
\begin{aligned}
q_{G H} \operatorname{Ker}\left(\alpha_{G}\right) & =\operatorname{Im}\left(q_{G H} \tau_{G} \operatorname{Tor}_{1}^{\mathbb{Z}}\left(i d, \rho^{H}\right)\right) \\
& =\operatorname{Im}\left(\left(\bar{i}^{\mathcal{G} H} \tau_{H}-q_{G H} \tau_{G}\right) \circ \operatorname{Tor}_{1}^{\mathbb{Z}}\left(i d, \rho^{H}\right)\right)
\end{aligned}
$$

since $\tau_{H} \circ \operatorname{Tor}_{1}^{\mathbb{Z}}\left(i d, \rho^{H}\right)=0$ as these are consecutive maps in the six term exact sequence part of which is displayed in (35). Thus by (48),

$$
\operatorname{Ker}\left(\bar{\alpha}_{G}\right)=\operatorname{Im}\left(\bar{i}^{\mathcal{G H H}} \tau_{H}-q_{G H} \tau_{G}\right)
$$


Now we are ready to compute $\nu_{21} \operatorname{Ker}\left(\beta_{G}\right)$. First of all we note that

$$
\nu_{21}\left(m_{2}^{\mathcal{G}} \otimes i d\right) i^{\mathcal{G H H}}\left(G^{A B} \otimes l_{2}^{H} \operatorname{Ker}\left(c_{2}^{H}\right)\right)=0
$$

since

$$
\nu_{21}\left(m_{2}^{\mathcal{G}} \otimes i d\right) i^{\mathcal{G} H H}=\nu_{12}\left(i d \otimes m_{2}^{H}\right)
$$

by middle associativity of the tensor product over $\mathrm{UL}(H)$ used to define $\mathrm{U}^{\mathcal{G}}(G, H)$. Hence $\nu_{21}$ factors as $\mathrm{U}_{2} \mathrm{~L}^{\mathcal{G}}(G) \otimes H^{a b} \stackrel{q_{G H}}{\longrightarrow} X \stackrel{\bar{\nu}_{21}}{\longrightarrow} \mathrm{U}_{3}^{\mathcal{G}}(G, H)$, and $\nu_{21} \operatorname{Ker}\left(\beta_{G}\right)=$ $\bar{\nu}_{21} \operatorname{Ker}\left(\bar{\alpha}_{G}\right)$. But $\bar{\nu}_{21} \overline{i^{\mathcal{G} H H}}=\nu_{12}$ since

$$
\begin{aligned}
\bar{\nu}_{21} \overline{i^{\mathcal{G} H}}\left(i d \otimes m_{2}^{H}\right) & =\bar{\nu}_{21} q_{G H}\left(m_{2}^{\mathcal{G}} \otimes i d\right) i^{\mathcal{G} H H} \\
& =\nu_{21}\left(m_{2}^{\mathcal{G}} \otimes i d\right) i^{\mathcal{G} H H} \\
& =\nu_{12}\left(i d \otimes m_{2}^{H}\right)
\end{aligned}
$$

again by (51). Hence

$$
\nu_{21} \operatorname{Ker}\left(\beta_{G}\right)=\operatorname{Im}\left(\nu_{12} \tau_{H}-\nu_{21} \tau_{G}\right)=\operatorname{Im}\left(\delta_{1}\right)
$$

as desired.

Step 2. Consider the following diagram.

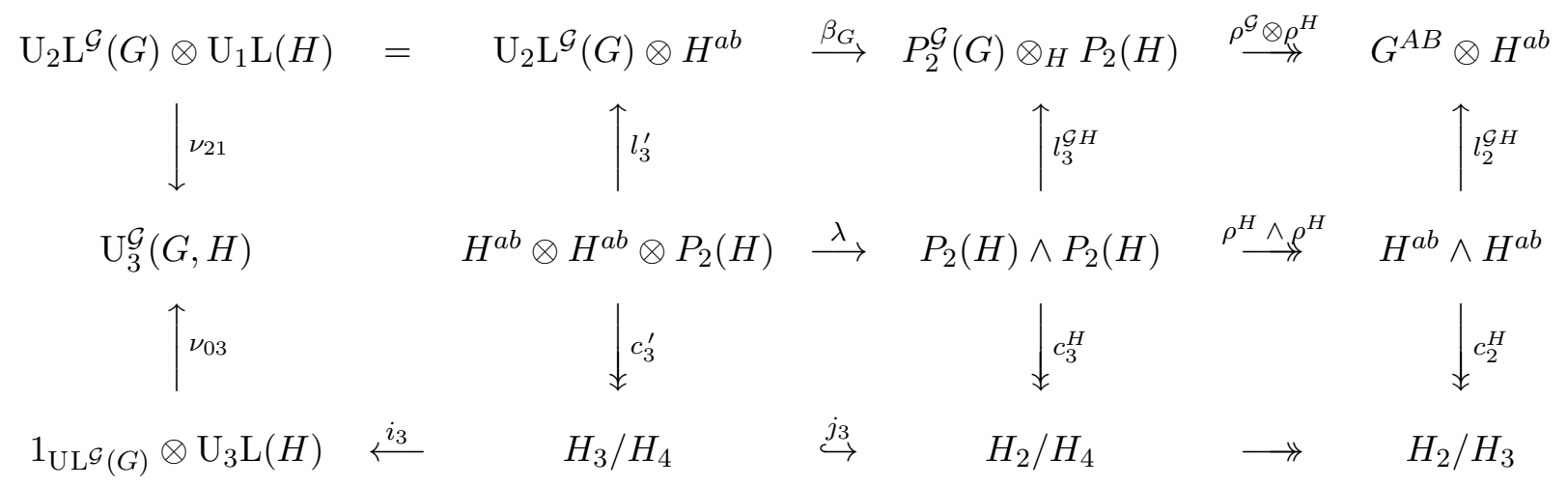

Here $l_{3}^{\prime}=\left(m_{2}^{\mathcal{G}} \otimes i d\right) i^{H H H} l_{33}^{H}\left(i d \otimes i d \otimes \rho^{H}\right), c_{3}^{\prime}=c_{33}^{H}\left(i d \otimes i d \otimes \rho^{H}\right), \lambda=q_{\wedge}\left(\mu_{2}^{H} m_{2}^{H} \otimes\right.$ $i d)$ with $q_{\wedge}: P_{2}(H) \otimes P_{2}(H) \rightarrow P_{2}(H) \wedge P_{2}(H)$, and $i_{3}: H_{3} / H_{4}=\mathrm{L}_{3}(H) \rightarrow$ $\mathrm{U}_{3} \mathrm{~L}(H) \rightarrow 1_{\mathrm{UL}^{\mathcal{G}}(G)} \otimes \mathrm{U}_{3} \mathrm{~L}(H)$ is the composite of the canonical maps.

Diagram (52) commutes; this is clear from the definitions for the two rightmost squares and was essentially proved in [4, Lemma 4.3] for the two middle squares. For the left hand rectangle this is due to middle associativity of the tensor product over $\mathrm{UL}(H)$ used to define $\mathrm{U}^{\mathcal{G}}(G, H)$. Moreover, omitting the left hand rectangle, the rows of the diagram are exact by (32), (33). Now define the additive relation $\delta_{2}: \operatorname{Ker}\left(c_{2}^{H}\right) \cap \operatorname{Ker}\left(l_{2}^{\mathcal{G} H}\right) \rightarrow \mathrm{U}_{3}^{\mathcal{G}}(G, H)$ by

$$
\delta_{2}=\left(\nu_{21} \beta_{G}^{-1} l_{3}^{\mathcal{G} H}-\nu_{03} i_{3} j_{3}^{-1} c_{3}^{H}\right)\left(\rho^{H} \wedge \rho^{H}\right)^{-1} .
$$


The indeterminacy of the first factor from the left is $\nu_{21} \operatorname{Ker}\left(\beta_{G}\right)=\operatorname{Im}\left(\delta_{1}\right)$ by (42), and the indeterminacy of the second factor is annihilated by the first modulo $\operatorname{Im}\left(\delta_{1}\right)$ by commutativity of diagram (52) and exactness of its middle row. Whence $\delta_{2}$ is a welldefined homomorphism modulo $\operatorname{Im}\left(\delta_{1}\right)$.

Let $q_{3}: \mathrm{U}_{3}^{\mathcal{G}}(G, H) \rightarrow \mathrm{U}_{3}^{\mathcal{G}}(G, H) / \operatorname{Im}\left(\delta_{1}\right)$ and define the homomorphism

$$
\delta_{2}^{\prime}: \operatorname{Ker}\left(c_{3}^{H}\right) \cap\left(l_{3}^{\mathcal{G} H}\right)^{-1} \operatorname{Im}\left(\beta_{G}\right) \longrightarrow \mathrm{U}_{3}^{\mathcal{G}}(G, H) / \operatorname{Im}\left(\delta_{1}\right), \quad \delta_{2}^{\prime}=q_{3} \nu_{21} \beta_{G}^{-1} l_{3}^{\mathcal{G} H} .
$$

By (41) one has $q_{3} \operatorname{Ker}\left(\theta_{3}^{\mathcal{G H}}\right)=\operatorname{Im}\left(\delta_{2}^{\prime}\right)$. Now by the snake lemma, diagram (52) induces an exact sequence

$$
\operatorname{Ker}\left(c_{3}^{\prime}\right) \longrightarrow \operatorname{Ker}\left(c_{3}^{H}\right) \longrightarrow \operatorname{Ker}\left(c_{2}^{H}\right) \longrightarrow \operatorname{Coker}\left(c_{3}^{\prime}\right)=0
$$

which implies another exact sequence

$$
\operatorname{Ker}\left(c_{3}^{\prime}\right) \stackrel{\lambda^{\prime}}{\longrightarrow} \operatorname{Ker}\left(c_{3}^{H}\right) \cap\left(l_{3}^{\mathcal{G} H}\right)^{-1} \operatorname{Im}\left(\beta_{G}\right) \stackrel{\rho^{\prime}}{\longrightarrow} \operatorname{Ker}\left(c_{2}^{H}\right) \cap \operatorname{Ker}\left(l_{2}^{\mathcal{G} H}\right) \rightarrow 0
$$

where $\lambda^{\prime}$ and $\rho^{\prime}$ are the restrictions of $\lambda$ and $\rho^{H} \wedge \rho^{H}$, resp. Now $\delta_{2}^{\prime} \lambda^{\prime}=q_{3} \nu_{21} l_{3}^{\prime}=$ $q_{3} \nu_{03} i_{3} c_{3}^{\prime}=0$, so $\delta_{2}^{\prime}$ factors as

$\operatorname{Ker}\left(c_{3}^{H}\right) \cap\left(l_{3}^{\mathcal{G} H}\right)^{-1} \operatorname{Im}\left(\beta_{G}\right) \stackrel{\rho^{\prime}}{\longrightarrow} \operatorname{Ker}\left(c_{2}^{H}\right) \cap \operatorname{Ker}\left(l_{2}^{\mathcal{G} H}\right) \stackrel{\bar{\delta}_{2}^{\prime}}{\longrightarrow} \mathrm{U}_{3}^{\mathcal{G}}(G, H) / \operatorname{Im}\left(\delta_{1}\right)$. Let $x \in \operatorname{Ker}\left(c_{2}^{H}\right) \cap \operatorname{Ker}\left(l_{2}^{\mathcal{G} H}\right)$. By (154) there is $x^{\prime} \in \operatorname{Ker}\left(c_{3}^{H}\right) \cap\left(l_{3}^{\mathcal{G} H}\right)^{-1} \operatorname{Im}\left(\beta_{G}\right)$ such that $\rho^{\prime}\left(x^{\prime}\right)=x$. Then $\bar{\delta}_{2}^{\prime}(x)=\delta_{2}^{\prime}\left(x^{\prime}\right)=q_{3}\left(\nu_{21} \beta_{G}^{-1} l_{3}^{\mathcal{G} H}-\nu_{03} i_{3} j_{3}^{-1} c_{3}^{H}\right)\left(x^{\prime}\right)=$ $q_{3} \delta_{2}(x)$. Thus $\bar{\delta}_{2}^{\prime}=q_{3} \delta_{2}$ and $q_{3} \operatorname{Ker}\left(\theta_{3}^{\mathcal{G H}}\right)=\operatorname{Im}\left(\delta_{2}^{\prime}\right)=\operatorname{Im}\left(\bar{\delta}_{2}^{\prime}\right)=q_{3} \operatorname{Im}\left(\delta_{2}\right)$ which proves relation (43).

Step 3. The explicit formula for $\delta_{2}$ in (38) is obtained by a straightforward calculation, taking $\sum_{1 \leq i<j \leq r} a_{i j} p_{2}\left(h_{i}\right) \wedge p_{2}\left(h_{j}\right)$ as a representative element of the coset $\left(\rho^{H} \wedge \rho^{H}\right)^{-1}(x)$, and using the following device: for $a, b \in G, p_{2}(a b)=p_{2}(a)+$ $p_{2}(b)+p_{2}(a) p_{2}(b)$; in particular, if $a$ or $b$ is in $G_{(2)}, p_{2}(a b)=p_{2}(a)+p_{2}(b)$. More generally, for $x_{1}, \ldots, x_{n} \in G, p_{2}\left(\prod_{i=1}^{n} x_{i}\right)=\sum_{i=1}^{n} p_{2}\left(x_{i}\right)+\sum_{1 \leq i<j \leq n} p_{2}\left(x_{i}\right) p_{2}\left(x_{j}\right)$. In particular, $p_{2}\left(x^{n}\right)=n p_{2}(x)+\left(\begin{array}{l}n \\ 2\end{array}\right) p_{2}(x)^{2}$ which is also true for negative $n$. This implies the equation

$$
d_{k} p_{2}\left(g_{k}\right) \otimes p_{2}\left(h_{k}\right)=p_{2}\left(g_{k}\right) \otimes\left(p_{2}\left(h_{k}^{d_{k}}\right)-\left(\begin{array}{c}
d_{k} \\
2
\end{array}\right) p_{k}\left(h_{k}\right)^{2}\right) .
$$

Finally, for $g \in G$ and $h^{\prime} \in H_{2}$,

$$
\nu_{21} \beta_{G}^{-1}\left(p_{2}(g) \otimes p_{2}\left(h^{\prime}\right)\right)=\nu_{12}\left(\left(g G_{(2)}\right) \otimes\left(h^{\prime} H_{3}\right)\right)+\operatorname{Im}\left(\delta_{1}\right)
$$

which is deduced from the following relations for $h_{1}, h_{2} \in H, h_{i}^{\prime} \in H_{2}$ :

$$
\begin{aligned}
p_{2}\left(\prod_{i} h_{i}^{\prime}\right) & =\sum_{i} p_{2}\left(h_{i}^{\prime}\right) \\
p_{2}\left(\left[h_{1}, h_{2}\right]\right) & =\left[p_{2}\left(h_{1}\right), p_{2}\left(h_{2}\right)\right] \\
\nu_{21} \beta_{G}^{-1}\left(p_{2}(g) \otimes p_{2}\left(h_{1}\right) p_{2}\left(h_{2}\right)\right) & =\nu_{21} \beta_{G}^{-1}\left(p_{2}(g) p_{2}\left(h_{1}\right) \otimes p_{2}\left(h_{2}\right)\right) \\
& =\left(g G_{(2)}\right) \cdot\left(h_{1} G_{(2)}\right) \otimes\left(h_{2} H_{2}\right)+\operatorname{Im}\left(\delta_{1}\right) \\
& =\left(g G_{(2)}\right) \otimes\left(h_{1} H_{2}\right) \cdot\left(h_{2} H_{2}\right)+\operatorname{Im}\left(\delta_{1}\right) \\
& =\nu_{12}\left(\left(g G_{(2)}\right) \otimes\left(h_{1} H_{2}\right) \cdot\left(h_{2} H_{2}\right)\right)+\operatorname{Im}\left(\delta_{1}\right)
\end{aligned}
$$


We now establish the link between the description of $\operatorname{Ker}\left(\theta_{3}^{\mathcal{G} H}\right)$ given by Theorem 6.1 and the subgroup $\mathcal{R}_{3}^{\mathcal{G} H}$ of $\operatorname{Ker}\left(\theta_{3}^{\mathcal{G} H}\right)$ constructed in section 5. First note that $\operatorname{Ker}\left(l_{2}^{\mathcal{G} H}\right)$ contains the canonical subgroup

$$
\Gamma=\operatorname{Im}\left(\left(\left(H \cap G_{(2)}\right) / H_{2}\right) \wedge\left(\left(H \cap G_{(2)}\right) / H_{2}\right) \rightarrow\left(H / H_{2}\right) \wedge\left(H / H_{2}\right)\right) .
$$

Lemma 6.7 One has $\delta_{2}\left(\Gamma \cap \operatorname{Ker}\left(c_{2}\right)\right) \equiv \mathcal{R}_{3}^{\mathcal{G} H} \bmod \operatorname{Im}\left(\delta_{1}\right)$.

Proof: Let $x \in \Gamma \cap \operatorname{Ker}\left(c_{2}\right)$. Then $x=\sum_{q=1}^{p}\left(h_{q} H_{2}\right) \wedge\left(h_{q}^{\prime} H_{2}\right)$ with $h_{q}, h_{q}^{\prime} \in$ $H \cap G_{(2)}, q=1, \ldots, p$, such that $h:=\prod_{q=1}^{p}\left[h_{q}, h_{q}^{\prime}\right] \in H_{3}$. First of all, note that $x^{\prime}=\sum_{q=1}^{p} p_{2}\left(h_{q}\right) \wedge p_{2}\left(h_{q}^{\prime}\right) \in\left(\rho^{H} \wedge \rho^{H}\right)^{-1}(x)$. Then using the fact that $\left[p_{2}\left(h_{q}\right), p_{2}\left(h_{q}^{\prime}\right)\right]=0$ in $P_{2}^{\mathcal{G}}(G)$ since $p_{2}\left(h_{q}\right) \in p_{2}\left(G_{(2)}\right) \subset Q_{2}^{\mathcal{G}}(G)$ we get

$$
\begin{aligned}
l_{3}^{\mathcal{G H} H}\left(x^{\prime}\right) & =\sum_{q=1}^{p}\left(p_{2}\left(h_{q}\right) \otimes p_{2}\left(h_{q}^{\prime}\right)-p_{2}\left(h_{q}^{\prime}\right) \otimes p_{2}\left(h_{q}\right)\right) \\
& =\beta_{G}\left(\sum_{q=1}^{p}\left(h_{q} G_{(3)}\right) \otimes\left(h_{q}^{\prime} H_{2}\right)-\left(h_{q}^{\prime} G_{(3)}\right) \otimes\left(h_{q} H_{2}\right)\right) .
\end{aligned}
$$

On the other hand,

$$
\nu_{03} i_{3} j_{3}^{-1} c_{3}^{H}\left(\rho^{H} \wedge \rho^{H}\right)^{-1}(x) \equiv \nu_{03} i_{3} j_{3}^{-1}\left(\prod_{q=1}^{p}\left[h_{q}, h_{q}^{\prime}\right] H_{4}\right)=\nu_{03} i_{3}\left(h H_{4}\right)=1 \otimes\left(h H_{4}\right)
$$

Therefore

$$
\begin{aligned}
\delta_{2}(x) & =\sum_{q=1}^{p}\left(\left(h_{q} G_{(3)}\right) \otimes\left(h_{q}^{\prime} H_{2}\right)-\left(h_{q}^{\prime} G_{(3)}\right) \otimes\left(h_{q} H_{2}\right)\right)-1 \otimes\left(h H_{4}\right)+\operatorname{Im}\left(\delta_{1}\right) \\
& =-R_{3}\left(\underline{h}_{1}, \ldots, \underline{h}_{p}\right)+\operatorname{Im}\left(\delta_{1}\right)
\end{aligned}
$$

where each $\underline{h}_{q}=\left(h_{q}, h_{q}^{\prime}\right)$ is of height $\geq n=3$, with $k=1$ and $l=2$ for $h_{q}$ and for $h_{q}^{\prime}$. The rest should now be clear.

Proof of Corollary 6.2; $\quad$ By Theorem 6.1, $\operatorname{Ker}\left(\bar{\theta}_{3}^{\mathcal{G}, H}\right)=q \operatorname{Im}\left(\delta_{1}, \delta_{2}\right)$ where $q: \mathrm{U}_{3}^{\mathcal{G}}(G, H) \rightarrow \overline{\mathrm{U}}_{3}(G, H)$ is the canonical projection. By Lemma 6.7, $\delta_{2}$ induces a homomorphism $\bar{\delta}_{2}: \frac{\operatorname{Ker}\left(l_{2}^{\mathcal{G} H}\right) \cap \operatorname{Ker}\left(c_{2}^{H}\right)}{\Gamma \cap \operatorname{Ker}\left(c_{2}^{H}\right)} \longrightarrow \frac{\overline{\mathrm{U}}_{3}(G, H)}{q \operatorname{Im}\left(\delta_{1}\right)}$ and we have an exact sequence $0 \rightarrow q \operatorname{Im}\left(\delta_{1}\right) \rightarrow q \operatorname{Im}\left(\delta_{1}, \delta_{2}\right) \rightarrow \operatorname{Im}\left(\bar{\delta}_{2}\right) \rightarrow 0$. But the quotient $\operatorname{Ker}\left(l_{2}^{\mathcal{G} H}\right) / \Gamma$ is torsion by [8, Lemma 2.7]. Hence its subgroup $\frac{\operatorname{Ker}\left(l_{2}^{\mathcal{G} H}\right) \cap \operatorname{Ker}\left(c_{2}^{H}\right)}{\Gamma \cap \operatorname{Ker}\left(c_{2}^{H}\right)}$ is torsion, too. Thus being an extension of torsion groups, $\operatorname{Ker}\left(\bar{\theta}_{3}^{\mathcal{G} H}\right)$ is torsion which implies the assertion. 
Proof of corollaries 6.3 and 6.5: $\quad$ One has the following sequence of homomorphisms

$\operatorname{Tor}_{1}^{\mathbb{Z}}\left(G^{A B}, G^{A B}\right) \stackrel{\tau_{1}}{\longrightarrow} G^{A B} \otimes\left(G_{(2)} / G_{2}\right) \stackrel{\bar{\nu}}{\longrightarrow} \frac{G^{a b} \wedge G^{a b}}{\operatorname{Im}\left(\left(G_{(2)} / G_{2}\right) \wedge\left(G_{(2)} / G_{2}\right)\right)} \stackrel{\overline{l_{2}^{\mathcal{G} G}}}{\longrightarrow} G^{A B} \otimes G^{a b}$

where $\bar{\nu}\left(g G_{(2)} \otimes g^{\prime} G_{2}\right)=\overline{g G_{2} \wedge g^{\prime} G_{2}}, g, g^{\prime} \in G$. Now consider the following commutative diagram where $\hat{c}_{2}^{G}, \check{c}_{2}^{G}$ are given by restriction of $c_{2}^{G}$.

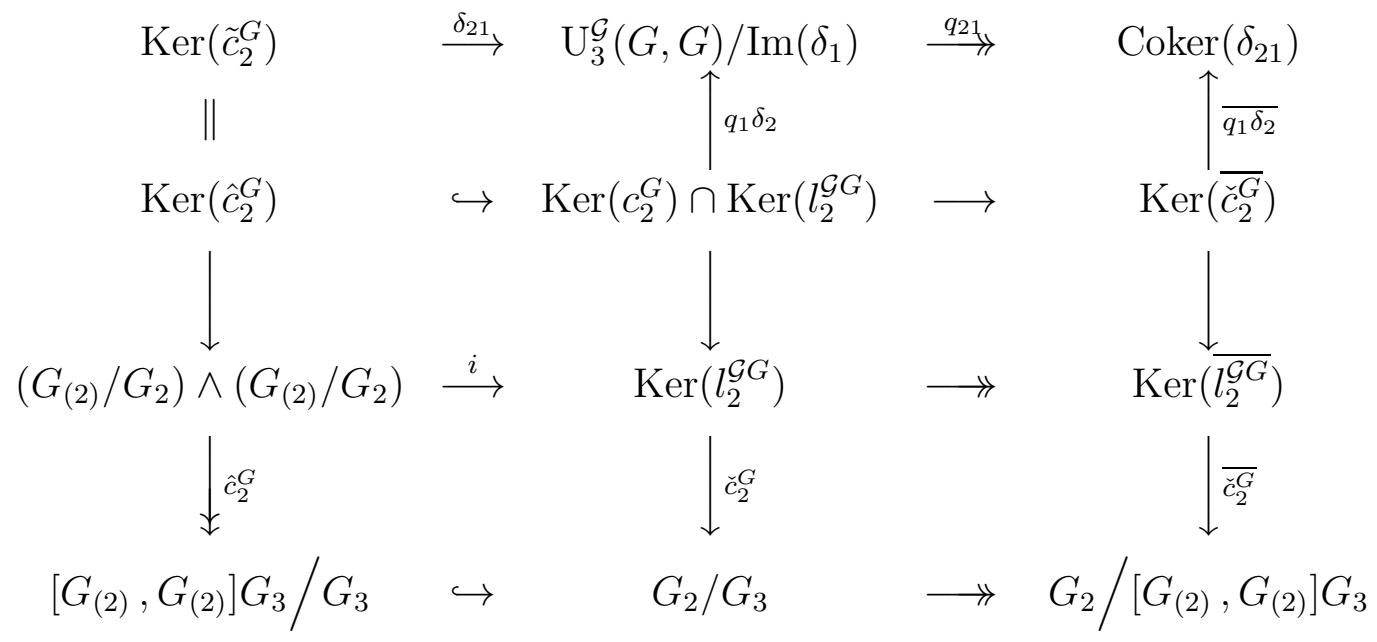

The columns and the two bottom rows are exact, so by the snake lemma the second row is short exact as $\hat{c}_{2}^{G}$ is surjective. Now by Lemma 2.7 in [8] one has $\operatorname{Ker}\left(\overline{l_{2}^{\mathcal{G} G}}\right)=\operatorname{Im}\left(\bar{\nu} \tau_{1}\right)$, so $\operatorname{Ker}\left(\overline{\check{c}_{2}^{G}}\right)=\bar{\nu} \tau_{1} \operatorname{Ker}\left(\overline{\check{c}_{2}^{G}} \bar{\nu} \tau_{1}\right)=\bar{\nu} \tau_{1} \operatorname{Ker}\left([,] \tau_{1}\right)$. So letting $\delta_{22}$ be the restriction of $\overline{q_{1} \delta_{2}} \bar{\nu} \tau_{1}$ to $\operatorname{Ker}\left([,] \tau_{1}\right)$ we have $\operatorname{Im}\left(\delta_{22}\right)=\operatorname{Im}\left(\overline{q_{1} \delta_{2}}\right)=$ $q_{21} \operatorname{Im}\left(q_{1} \delta_{2}\right)=q_{21} q_{1} \operatorname{Ker}\left(\theta_{3}^{\mathcal{G} G}\right)$ by Theorem 6.1, whence Corollary 6.3 is proved. As to Corollary 6.5 first note that by (37), $U_{1}=\operatorname{Im}\left(\delta_{1}\right)$. Now let $x \in \operatorname{Ker}\left(l_{2}^{\mathcal{G} G}\right)$. By the identity $\operatorname{Ker}\left(\overline{l_{2}^{\mathcal{G G}}}\right)=\operatorname{Im}\left(\bar{\nu} \tau_{1}\right)$ above there is $y \in \operatorname{Tor}_{1}^{\mathbb{Z}}\left(G^{A B}, G^{A B}\right)$ such that $\bar{x}=\bar{\nu} \tau_{1}(y)$ in $G^{a b} \wedge G^{a b} / \operatorname{Im}\left(\left(G_{(2)} / G_{2}\right) \wedge\left(G_{(2)} / G_{2}\right)\right)$. By [19] V.6, $y=$ $\sum_{r=1}^{s}\left\langle c_{r} G_{(2)}, k_{r}, d_{r} G_{(2)}\right\rangle$ with $s \geq 1, c_{r}, d_{r} \in G, k_{r} \in \mathbb{Z}$ such that $c_{r}^{k_{r}}, d_{r}^{k_{r}} \in$ $G_{(2)}$, and $\bar{\nu} \tau_{1}(y)=\sum_{r=1}^{s} \overline{\left(c_{r} G_{2}\right) \wedge\left(d_{r}^{k_{r}} G_{2}\right)}$. Thus $x=\sum_{q=1}^{p}\left(a_{q} G_{2}\right) \wedge\left(b_{q} G_{2}\right)+$ $\sum_{r=1}^{s}\left(c_{r} G_{2}\right) \wedge\left(d_{r}^{k_{r}} G_{2}\right)$ with $q \geq 1$ and $a_{q}, b_{q} \in G_{(2)}$. Now suppose that $x \in \operatorname{Ker}\left(c_{2}^{G}\right)$ which means that $g=\prod_{q=1}^{p}\left[a_{q}, b_{q}\right] \prod_{r=1}^{s}\left[c_{r}, d_{r}^{k_{r}}\right] \in G_{3}$. To compute $\delta_{2}(x)$ first note that putting $x_{1}=\sum_{q=1}^{p} p_{2}\left(a_{q}\right) \wedge p_{2}\left(b_{q}\right)$ and $x_{2}=\sum_{r=1}^{s} p_{2}\left(c_{r}\right) \wedge p_{2}\left(d_{r}^{k_{r}}\right)$ one has $x_{1}+x_{2} \in\left(\rho^{G} \wedge \rho^{G}\right)^{-1}(x)$. By the calculation in the proof of Lemma 6.7,

$$
l_{3}^{\mathcal{G} G}\left(x_{1}\right)=\beta_{G}\left(\sum_{q=1}^{p}\left(a_{q} G_{(3)}\right) \otimes\left(b_{q} G_{2}\right)-\left(b_{q} G_{(3)}\right) \otimes\left(a_{q} G_{2}\right)\right) .
$$

Now note that $\left[p_{2}\left(c_{r}\right), p_{2}\left(d_{r}^{k_{r}}\right)\right]=p_{2}\left(\left[c_{r}, d_{r}^{k_{r}}\right]\right)=0$ in $P_{2}^{\mathcal{G}}(G)$ since $\left[c_{r}, d_{r}^{k_{r}}\right] \in G_{(3)}$. This justifies the first of the following identities. 


$$
\begin{aligned}
l_{3}^{\mathcal{G} G}\left(x_{2}\right)= & \sum_{r=1}^{s}\left(p_{2}\left(c_{r}\right) \otimes p_{2}\left(d_{r}^{k_{r}}\right)-p_{2}\left(d_{r}^{k_{r}}\right) \otimes p_{2}\left(c_{r}\right)\right) \\
= & \sum_{r=1}^{s}\left(p_{2}\left(c_{r}\right) \otimes\left(k_{r} p_{2}\left(d_{r}\right)+\left(\begin{array}{c}
k_{r} \\
2
\end{array}\right) p_{2}\left(d_{r}\right)^{2}\right)-p_{2}\left(d_{r}^{k_{r}}\right) \otimes p_{2}\left(c_{r}\right)\right) \\
= & \sum_{r=1}^{s}\left(k_{r} p_{2}\left(c_{r}\right) \otimes p_{2}\left(d_{r}\right)+\left(\begin{array}{c}
k_{r} \\
2
\end{array}\right) p_{2}\left(c_{r}\right) \otimes p_{2}\left(d_{r}\right)^{2}-p_{2}\left(d_{r}^{k_{r}}\right) \otimes p_{2}\left(c_{r}\right)\right) \\
= & \sum_{r=1}^{s}\left(\left(p_{2}\left(c_{r}^{k_{r}}\right)-\left(\begin{array}{c}
k_{r} \\
2
\end{array}\right) p_{2}\left(c_{r}\right)^{2}\right) \otimes p_{2}\left(d_{r}\right)+\left(\begin{array}{c}
k_{r} \\
2
\end{array}\right) p_{2}\left(c_{r}\right) p_{2}\left(d_{r}\right) \otimes p_{2}\left(d_{r}\right)\right. \\
& \left.-p_{2}\left(d_{r}^{k_{r}}\right) \otimes p_{2}\left(c_{r}\right)\right) \\
= & \sum_{r=1}^{s}\left(p_{2}\left(c_{r}^{k_{r}}\right) \otimes p_{2}\left(d_{r}\right)-p_{2}\left(d_{r}^{k_{r}}\right) \otimes p_{2}\left(c_{r}\right)\right. \\
& \left.+\left(\begin{array}{c}
k_{r} \\
2
\end{array}\right) p_{2}\left(c_{r}\right)\left(p_{2}\left(d_{r}\right)-p_{2}\left(c_{r}\right)\right) \otimes p_{2}\left(d_{r}\right)\right) \\
= & \beta_{G}\left(\sum _ { r = 1 } ^ { s } \left(\left(c_{r}^{k_{r}} G_{(3)}\right) \otimes\left(d_{r} G_{2}\right)-\left(d_{r}^{k_{r}} G_{(3)}\right) \otimes\left(c_{r} G_{2}\right)\right.\right. \\
& \left.\left.+\left(\begin{array}{c}
k_{r} \\
2
\end{array}\right)\left(\left(c_{r} G_{(2)}\right)\left(\left(d_{r} G_{(2)}\right)-\left(c_{r} G_{(2)}\right)\right) \otimes\left(d_{r} G_{2}\right)\right)\right)\right)
\end{aligned}
$$

It now follows from (55) and (56) that $\delta_{2}(x)$ is represented modulo $\operatorname{Im}\left(\delta_{1}\right)$ by the element given in (31) which achieves the proof.

\section{ACKNOWLEDGMENTS}

The author is indebted to P. Littelmann for a helpful discussion about higher commutators. Furthermore, he wishes to express his gratitude to the Institut de Recherche Mathématique Avancée, Strasbourg, and in particular to his former director, Jean-Louis Loday, for the warm hospitality and ideal working conditions provided during the author's postdoctoral fellowship in 1993-1995 where part of the research presented here was done.

\section{References}

[1] F. Bachmann, L. Gruenenfelder, Über Lie-Ringe von Gruppen und ihre universellen Enveloppen, Comment. Math. Helv. 47 (1972) 332-340.

[2] F. Bachmann, L. Gruenenfelder, Homological methods and the third dimension subgroup, Comment. Math. Helv. 47 (1972) 526-531. 
[3] N. Gupta, Free group rings, Contemporary Mathematics, Vol. 66, American Mathematical Society, 1987.

[4] M. Hartl, Some successive quotients of group ring filtrations induced by Nseries, Comm. in Algebra 23 (10) (1995) 3831-3853.

[5] M. Hartl, Polynomiality properties of group extensions with torsion-free abelian kernel, J. of Algebra 179 (1996) 380-415.

[6] M. Hartl, The nonabelian tensor square and Schur multiplicator of nilpotent groups of class 2, J. of Algebra 179 (1996) 416-440.

[7] M. Hartl, A universal coefficient decomposition for subgroups induced by submodules of group algebras, Canadian Math. Bull. 40 (1) (1997) 47-53.

[8] M. Hartl, The relative second Fox and third dimension subgroup of arbitrary groups, Indian J. Pure Appl. Math. 39 (5) (2008), 435-451.

[9] M. Hartl, On Fox and augmentation quotients of semidirect products, under revision for J. of Algebra, http://arxiv.org/abs/0707.0362.

[10] M. Hartl, R. Mikhailov, I. B. S. Passi, Dimension quotients, J. Indian Math. Soc. (N.S.) 2007, Special volume on the occasion of the centenary year of IMS (1907-2007), 63-107 (2008).

[11] B. Hartley, The residual nilpotence of wreath products, Proc. London Math. Soc. (3) 20 (1970) 365-392.

[12] P.J. Hilton, U. Stammbach, A Course in Homological Algebra, Graduate Texts in Math. Vol. 4, Springer-Verlag, Berlin, Heidelberg, New York, 1971.

[13] R. Karan, L.R. Vermani, A note on augmentation quotients, Bull. London Math. Soc. 18 (1986) 5-6.

[14] R. Karan, L.R. Vermani, Augmentation quotients of integral group rings, J. Indian Math. Soc. 54 (1989) 107-120.

[15] R. Karan, L.R. Vermani, Augmentation quotients of integral group rings-II, J. Pure Appl. Algebra 65 (1990) 253-262.

[16] M. Khambadkone, Subgroup ideals in group rings I, J. Pure Appl. Algebra 30 (1983) 261-275.

[17] M. Khambadkone, On the structure of augmentation ideals in group rings, $J$. Pure Appl. Algebra 35 (1985) 35-45.

[18] M. Khambadkone, Augmentation quotients of semidirect products, Arch. Math. 45 (1985) 407-420. 
[19] S. Mac Lane, Homology, Springer Grundlehren, Vol. 114, Springer-Verlag Berlin-Göttingen-Heidelberg, 1963.

[20] I.B.S. Passi, Polynomial maps on groups, J. Algebra 9 (1968) 121-151.

[21] I.B.S. Passi, Group Rings and Their Augmentation Ideals, Lecture Notes in Math., Vol. 715, Springer-Verlag, Berlin, Heidelberg, New York, 1979.

[22] D. Quillen, On the associated graded of a group ring, J. Algebra 10 (1968) 411-418.

[23] R. Sandling, Note on the integral group ring problem, Math. Z. 124 (1972) $255-258$.

[24] K.-I. Tahara, Augmentation quotients and dimension subgroups of semidirect products, Math. Proc. Camb. Phil. Soc. 91 (1982), 39-49.

[25] K.I. Tahara, L.R. Vermani, Atul Razdan, On generalized third dimension subgroups, Canad. Math. Bull. 41 (1) (1998) 109-117.

[26] J. J. Rotman, An Introduction to Homological Algebra, Academic Press, Inc., 1979.

[27] A. Whitcomb, The group ring problem, Thesis, University of Chicago, March 1968. 